\title{
Microbial community dynamics in mesophilic and thermophilic batch reactors under methanogenic, phenyl acid-forming conditions
}

\author{
Eva Maria Prem ${ }^{1^{*}} \mathbb{D}$, Blaz Stres ${ }^{2,3,4}$, Paul IIImer ${ }^{1}$ and Andreas Otto Wagner ${ }^{1}$
}

\begin{abstract}
Background: Proteinaceous wastes exhibit high theoretical methane yields and their residues are considered valuable fertilisers. The routine anaerobic degradation of proteins often raises problems like high aromatic compound concentrations caused by the entry of aromatic amino acids into the system. A profound investigation of the consequences of aromatic compound exposure on various microorganisms, which cascade-like and interdependently degrade complex molecules to biogas, is still pending.
\end{abstract}

Results: In mesophilic samples, methane was predominantly produced via acetoclastic methanogenesis. The highest positive correlation was observed between phenylacetate (PAA) and Psychrobacter spp. and between phenylpropionate (PPA) and Haloimpatiens spp. Moreover, Syntrophus spp. negatively correlated with PAA (Spearman's rank correlations coefficient $(r s)=-0.46, p<0.05)$ and PPA concentrations ( $r s=-0.44, p<0.05)$ and was also associated with anaerobic benzene ring cleavage. In thermophilic samples, acetate was predominantly oxidised by Tepidanaerobacter spp. or Syntrophaceticus spp. in syntrophic association with a hydrogenotrophic methanogen. The genera Sedimentibacter and Syntrophaceticus correlated positively with both PAA and PPA concentrations. Moreover, Sedimentibacter spp., Tepidanaerobacter spp., Acetomicrobium spp., and Sporanaerobacter spp. were significant LEfSe (linear discriminant analysis effect size) biomarkers for high meso- as well as thermophilic phenyl acid concentrations. Direct negative effects of phenyl acids on methanogenic properties could not be proven.

Conclusions: Anaerobic phenyl acid formation is not restricted to specific microbial taxa, but rather done by various meso- and thermophilic bacteria. The cleavage of the highly inert benzene ring is possible in methanogenic batch reactors - at least in mesophilic fermentation processes. The results indicated that phenyl acids rather affect microorganisms engaged in preceding degradation steps than the ones involved in methanogenesis.

Keywords: Anaerobic digestion, Phenylacetate, Phenylpropionate, Aromatic compounds, Biogas, Next-generation sequencing, Piphillin analyses

*Correspondence: eva.prem@uibk.ac.at

1 Department of Microbiology, Universität Innsbruck, Technikerstraße 25d, 6020 Innsbruck, Austria

Full list of author information is available at the end of the article

\begin{abstract}
Background
On a global scale, waste products coming from food industry or from agriculture are available in large quantities. Using waste products of the respective region for biogas formation can be an economically effective and sustainable way to contribute to the renewable energy pool $[1,2]$. Biogas reactors rely on cascade-like
\end{abstract}


interactions between various microorganisms that interdependently degrade complex substrates to methane and carbon dioxide [3]. However, an increased use of waste products can be challenging due to undesirable compounds entering biogas plants [4-7]. Protein-rich waste products like slaughterhouse waste, thin stillage, or pig manure have indeed a high theoretical methane yield [8-10] and the resulting residues are considered desirable fertilisers [11]. However, the anaerobic degradation of proteins is often problematic due to the rise of ammonia $[8,11,12]$ or hydrogen sulphide [10]. Free ammonia is particularly toxic to acetoclastic methanogens; therefore, syntrophic acetate oxidation (SAO) combined with hydrogenotrophic methanogenesis is a common pathway in ammonia-rich anaerobic reactors [8-10].

Aromatic compounds are another group of potentially problematic materials [7, 13-16]. They are one of the most abundant organic compounds on earth and enter the biogas reactors via proteins, lignocellulosic materials, and pollutants [17]. Tryptophan (Tryp), tyrosine (Tyr), and phenylalanine (Phe) are aromatic amino acids thus contain a benzene ring, which is very stable due to its sixcarbon-joined planar ring structure [16]. They enter the biogas reactor via proteins, depending on the respective composition of the substrate [18].

Despite the ubiquitous occurrence of aromatic compounds, only microorganisms (prokaryotes and fungi) are able to completely degrade these materials [19]. Since the 1980s, several studies showed that not only aerobic, but also anaerobic benzene degradation is possible under certain electron accepting conditions (for example under methanogenic or sulphate-reducing conditions) [20-22]. The anaerobic degradation of aromatic compoundsalbeit considered distinctly slower than the aerobic approach-plays an important role in biogeochemical cycles as aromatic compounds are present in abundance in various anoxic habitats $[23,24]$. The phenyl acids phenylacetate (PAA) and phenylpropionate (PPA), two monocyclic aromatic acids, are relevant aromatic intermediates in the anaerobic degradation of benzenes $[4,7$, 25]; however, these two compounds received little attention so far [15]. Anaerobic Tyr and Phe degradation by fermenting bacteria was shown to lead to the formation of PAA and 4-hydroxyphenylacetate, respectively [23]. Some Clostridia were shown to degrade Phe to phenyllactate (PLA) and subsequently to PAA without attacking the benzene ring itself $[23,26]$. One key enzyme in the Phe degradation is the phenylacetaldehyde dehydrogenase responsible for the conversion of phenylacetaldehyde to PAA as shown with the model organisms Aromatoleum aromaticum and Thauera aromatica [27, 28].
Depending on the respective substituents, aromatic compounds are further anaerobically degraded via special central intermediates [19]. PAA and PPA are degraded to the intermediate benzoyl-CoA [23]. Once formed, benzoyl-CoA enters the central pathway leading to the de-aromatisation and (hydrolytic) cleavage of the phenyl ring [14, 23, 29-31]; albeit facultative and obligate anaerobic microorganisms use different enzymes during the benzoyl-CoA reduction [29]. In Thauera aromatica, benzoyl-CoA is further reduced to cyclohexa-1,5-diene1 -carbonyl-CoA by a benzoyl-CoA reductase. The next steps include a hydratase and a dehydrogenase. Ring cleavage finally takes place by adding $\mathrm{H}_{2} \mathrm{O}$ to the double bound of 6-oxocyclohex-1-ene-1-carbonyl-CoA by 6-oxocyclohex-1-ene-1-carbonyl-CoA hydrolase (Kyoto Encyclopedia of Genes and Genomes (KEGG) orthology K07539), which results in the formation of 3-hydroxypimelyl-CoA [29]. Anaerobic benzoate degradation via benzoyl-CoA has also been profoundly studied in model organisms other than Thauera aromatica, like Azoarcus spp. or Geobacter metallireducens [23, 28, 32-36]. Some model organisms are able to carry out several degradation steps within the respective peripheral and/or central pathway [27, 33]. Under more natural conditions, due to the complex microbial interactions and interdependencies, it is more likely that a variety of microbial species take part in the degradation of aromatic compounds [31]. By contrast, tryptophan is characterised by an indole ring system and is anaerobically degraded to 2 -aminobenzoylCoA using enzymes like 2-aminobenzoate-CoA ligase [23].

The effects of aromatic compounds on microorganisms in methanogenic communities are still not clear due to the previous use of different aromatic compounds, temperature regimes, and inocula. For instance, a single PAA pulse was shown to be responsible for an archaeal shift from acetoclastic to hydrogenotrophic methanogens in primary sludge digesters at mesophilic temperatures, whereas the archaeal communities were more stable in digesters containing primary/secondary sludge mixtures [4]. Moreover, PAA concentrations above $0.5 \mathrm{~g} \mathrm{~L}^{-1}$ led to clear inhibitory responses in thermophilic bioreactors [37]. By contrast, PAA and PPA were shown to have a stimulatory effect on the cellulose-degrader Ruminococcus albus $[38,39]$.

Wagner et al. [15] simulated different stages of overload using mesophilic and thermophilic batch communities and evaluated phenyl acid generation (PAA and PPA) and biogas production performance. Phenyl acid formation could be observed at certain overload conditions. PAA and PPA did not necessarily lead to a low methane generation $[5,15]$. Substrate load rather than temperature or inoculum was shown to influence PAA and PPA 
turnover [15]. In the present study, samples derived from this data set [15] were subjected to microbiological analysis in order (i) to give an overview of microbial shifts during anaerobic digestion (AD) of amino acids and proteinaceous substrates under different overload and temperature conditions; (ii) to investigate microorganisms involved in methanogenesis in more detail; (iii) to correlate the formation and degradation of phenyl acids to specific genera/microbial groups, and (iv) to search for general peripheral as well as central benzoyl-CoA pathways and for microbial enzymes associated with anaerobic cleavage of the benzene ring.

\section{Results}

\section{Mesophilic and thermophilic community composition}

Prior to filtering, 1661 operational taxonomic units (OTUs) were generated in total. Consequently, and irrespective of the overload conditions, the microbial diversity (Shannon Index) was considerably higher in mesophilic than in thermophilic samples as shown in Additional file 1: Fig. S3. Therefore, data were thenceforward analysed separately. To remove noisy OTU categories, OTUs with a total abundance below 10 were excluded from each temperature regime (abundance per sample of removed OTUs: $\leq 5)$. Thereafter, 659 OTUs and 282 OTUs remained for further analyses in mesophilic and thermophilic samples, respectively.

In total, 38 bacterial and five archaeal phyla were found in mesophilic samples. The most abundant mesophilic phyla were Bacteroidetes, Firmicutes, and Chloroflexi. The most abundant phylum in Tryp, Tyr, and control (Cont) samples was Bacteroidetes with a mean sequence abundance of $28 \%$ in Tryp and $29 \%$ in both Tyr and Cont samples. In Cas and ME samples, Firmicutes was the dominant phylum with a mean sequence abundance of 39\% (Cas) and 42\% (ME). In Phe samples, the contribution of Bacteroidetes and Firmicutes was balanced (25\% Firmicutes and $24 \%$ Bacteroidetes). The relative sequence abundance of the phylum Chloroflexi was highest in the high PAA concentration group (low: 14\%, medium: $12 \%$, and high: 22\%). By contrast, the abundance of Bacteroidetes was lower at higher PAA concentrations (low: 26\%, medium: 23\%, and high: 19\%). The phylum Firmicutes dominated at high PPA concentrations (low: 26\%, medium: $45 \%$, and high: $55 \%$ ). Significant phyla with an effect size $\geq 1$ are depicted in Additional file 1: Fig. S1 for low and high PAA and PPA concentrations. A comprehensive overview of mesophilic communities can be looked up in the respective KRONA file (Additional file 1: Fig. S4).

In contrast to mesophilic samples, only 19 bacterial and two archaeal phyla were associated with thermophilic samples. The phyla Thermotogae and Firmicutes dominated the thermophilic communities. The mean relative sequence abundance of Thermotogae (all sequences of this phylum were classified as genus Defluviitoga) was especially high in amino acid samples (Tryp: 61\%, Tyr: 54\%, and Phe: 60\%). The abundance of the phylum Firmicutes was highest in complex protein samples (ME: 57\% and Cas: 56\%). In the high PAA concentration group, the phylum Firmicutes was prevailing (relative abundance: 55\%), whereas Thermotogae was dominant in the medium PAA concentration group (55\%). The abundance of the phylum Synergistetes was relatively high at elevated PAA and PPA levels. Phyla with an effect size $\geq 1$ for low and high PAA and PPA concentration are depicted in Additional file 1: Fig. S2. A comprehensive overview of thermophilic communities can be found in the respective KRONA file (Additional file 1: Fig. S5).

\section{Mesophilic communities \\ Core microbiome and metagenomic biomarkers}

Core members for each substrate are listed in Table 1. The genera ADurb.Bin120 (Anaerolineaceae), Anaerolineaceae (uncultured genus), Bacteroidetes_vadinHA17_ genus, and Fastidiosipila were part of each mesophilic core microbiome, irrespective of the substrate or variation. The acetoclastic methanogen Methanosaeta was a core member of the control and Phe samples; no other methanogen could be detected in any mesophilic core microbiome.

Significant biomarkers with a linear discriminant analysis (LDA) score $\geq 4$ are listed in Table 2 for all substrates. The Cont, Tryp, and Tyr samples showed considerably more metagenomic biomarkers than Phe, ME, and Cas samples. Via the LEfSe (linear discriminant analysis effect size) algorithm using the substrate as class and the degree of overload (low, medium, high) as subclass, Methanoculleus spp. was shown to be a significant biomarker for Cas samples. Only few mesophilic microorganisms were core members as well as significant biomarkers: Methanosaeta and Candidatus_Cloacimonas for the controls, Proteiniphilum for Tryp samples, and Christensenellaceae_R-7_ group for Tyr samples (Tables 1 and 2).

\section{Phenyl acids and community dynamics}

Results of mesophilic phenyl acid formation were published previously [15] and are depicted in a summarised form in Additional file 1: Fig. S6. During mesophilic incubation, the controls did not form any phenyl acids, whereas all reactors containing additional substrates showed high phenyl acid concentrations. After 28 days, the highest PAA concentrations were found in Phe samples under medium load conditions; the highest PPA concentrations were detected in casein-fed reactors under high load conditions [15]. 
Table 1 List of genera defining the core microbiome of each substrate over all time points

\begin{tabular}{|c|c|c|c|c|}
\hline \multirow[t]{2}{*}{ Substrate } & \multicolumn{2}{|l|}{ Mesophilic } & \multicolumn{2}{|l|}{ Thermophilic } \\
\hline & Sample size & Core microbiome & Sample size & Core microbiome \\
\hline Cont & 9 & $\begin{array}{l}\text { ADurb.Bin120 (Anaerolineaceae) } \\
\text { Anaerolineaceae (uncultured) } \\
\text { Macellibacteroides } \\
\text { Proteiniphilum } \\
\text { Bacteroidetes_vadinHA17 } \\
\text { Candidatus Cloacimonas } \\
\text { Fastidiosipila } \\
\text { Methanosaeta } \\
\text { Synergistaceae (uncultured) } \\
\text { Cloacimonadaceae_W5 }\end{array}$ & 8 & $\begin{array}{l}\text { Defluviitoga } a^{b} \\
\text { Caldicoprobacter } \\
\text { DTU014 (Clostridia) } \\
\text { MBA03 (Clostridia) } \\
\text { Firmicutes (uncultured) }\end{array}$ \\
\hline Tryp & 12 & $\begin{array}{l}\text { ADurb.Bin120 (Anaerolineaceae) } \\
\text { Anaerolineaceae (uncultured) } \\
\text { Macellibacteroides } \\
\text { Proteiniphilum } \\
\text { Bacteroidetes_vadinHA17 } \\
\text { Fastidiosipila }^{\mathrm{a}} \\
\text { Synergistaceae (uncultured_genus 1) }\end{array}$ & 12 & $\begin{array}{l}\text { Defluviitoga }^{\mathrm{b}} \\
\text { Caldicoprobacter }^{\mathrm{b}} \\
\text { DTU014 (Clostridia) } \\
\text { MBA03 (Clostridia) } \\
\text { Syntrophaceticus }\end{array}$ \\
\hline Tyr & 12 & $\begin{array}{l}\text { ADurb.Bin120 (Anaerolineaceae) } \\
\text { Anaerolineaceae (uncultured) } \\
\text { Macellibacteroides } \\
\text { Proteiniphilum } \\
\text { Bacteroidetes_vadinHA17ª } \\
\text { Fastidiosipila }^{\mathrm{a}} \\
\text { Christensenellaceae_R-7_group } \\
\text { Synergistaceae (uncultured_genus 1) }\end{array}$ & 12 & $\begin{array}{l}\text { Defluviitoga } \\
\text { Caldicoprobacter } \\
\text { DTU014 (Clostridia) } \\
\text { Ruminiclostridium } \\
\text { Syntrophaceticus }\end{array}$ \\
\hline Phe & 12 & $\begin{array}{l}\text { ADurb.Bin120 (Anaerolineaceae) } \\
\text { Anaerolineaceae (uncultured) } \\
\text { Proteiniphilum } \\
\text { Bacteroidetes_vadinHA17 } \\
\text { Candidatus Cloacimonas } \\
\text { Fastidiosipila } \\
\text { Methanosaeta }\end{array}$ & 12 & $\begin{array}{l}\text { Defluviitoga }{ }^{b} \\
\text { DTU014 (Clostridia) } \\
\text { Syntrophaceticus }\end{array}$ \\
\hline ME & 18 & $\begin{array}{l}\text { ADurb.Bin120 (Anaerolineaceae) } \\
\text { Anaerolineaceae (uncultured) } \\
\text { Bacteroidetes_vadinHA17 } \\
\text { Candidatus Cloacimonas } \\
\text { Fastidiosipila }\end{array}$ & 17 & $\begin{array}{l}\text { Defluviitogab } \\
\text { Caldicoprobacter } \\
\text { DTU014 (Clostridia) } \\
\text { Proteiniphilum } \\
\text { Tepidanaerobacter } \\
\text { Sporanaerobacter } \\
\text { MBA03 (Clostridia) }\end{array}$ \\
\hline Cas & 18 & $\begin{array}{l}\text { ADurb.Bin120 (Anaerolineaceae) } \\
\text { Anaerolineaceae (uncultured) } \\
\text { Proteiniphilum } \\
\text { Bacteroidetes_vadinHA17 } \\
\text { Candidatus Cloacimonas } \\
\text { Fastidiosipila } \\
\text { Sedimentibacter } \\
\text { Ruminococcaceae (uncultured) }\end{array}$ & 18 & $\begin{array}{l}\text { Defluviitoga } \\
\text { Caldicoprobacter }^{\mathrm{b}} \\
\text { DTU014 (Clostridia) } \\
\text { Tepidanaerobacter } \\
\text { MBA03 (Clostridia) } \\
\text { Gelria }\end{array}$ \\
\hline
\end{tabular}

Genera marked with ${ }^{\mathrm{a}}$ and ${ }^{\mathrm{b}}$ were part of every mesophilic and thermophilic core microbiome, respectively

Spearman correlations (Benjamini-Hochberg $(\mathrm{B}-\mathrm{H})$ adjusted) were calculated for samples of day 28. More meso- than thermophilic taxa correlated $(p<0.05)$ with phenyl acid concentrations. Spearman rank correlation coefficients were also higher in meso- than in thermophilic samples. The highest positive $(p<0.05)$ correlations between PAA concentration and microbial genera could be shown with Psychrobacter, Rhizobiaceae (uncultured genus), and Candidatus_Symbiobacter (Fig. 1). Furthermore, PAA concentration was negatively $(p<0.05)$ correlated with several genera including W5 (Cloacimonadaceae), WCHB1-41 (Kiritimatiellae), and Ruminiclostridium (Fig. 1).

PPA concentration correlated highly positive $(p<0.05)$ with several mesophilic genera including Haloimpatiens, Proteus, and Tepidimicrobium (Fig. 1). Negative 
Table 2 Significant LEfSe biomarkers with a linear discriminant analysis (LDA) score $\geq 4$, using the respective substrate as class and the degree of overload (low, medium, high) as subclass

\begin{tabular}{|c|c|c|c|c|}
\hline \multirow[t]{2}{*}{ Substrate (class) } & \multicolumn{2}{|l|}{ Mesophilic } & \multicolumn{2}{|l|}{ Thermophilic } \\
\hline & Sample size & LEfSe biomarkers & Sample size & LEfSe biomarkers \\
\hline Cont & 9 & $\begin{array}{l}\text { Candidatus Cloacimonas } \\
\text { Methanosaeta } \\
\text { Pedosphaeraceae_genus } \\
\text { Gracilibacter }\end{array}$ & 8 & $\begin{array}{l}\text { Lachnospiraceae } \\
\text { (uncultured } \\
\text { genus) } \\
\text { Halocella }\end{array}$ \\
\hline Tryp & 6 & $\begin{array}{l}\text { Proteiniphilum } \\
\text { Desulfitobacterium }\end{array}$ & 6 & - \\
\hline Tyr & 6 & $\begin{array}{l}\text { Christensenellaceae_R-7_group } \\
\text { Lachnoclostridium_5 } \\
\text { Treponema_2 } \\
\text { Methanobacterium }\end{array}$ & 6 & Tepidimicrobium \\
\hline Phe & 6 & - & 6 & - \\
\hline ME & 6 & Paraclostridium & 5 & $\begin{array}{l}\text { Sporanaerobacter } \\
\text { Proteiniphilum }\end{array}$ \\
\hline Cas & 6 & $\begin{array}{l}\text { Romboutsia } \\
\text { Methanoculleus }\end{array}$ & 6 & $\begin{array}{l}\text { Gelria } \\
\text { Tepidanaerobacter }\end{array}$ \\
\hline
\end{tabular}

Sample sizes refer to each substrate-overload combination over all time measuring points for each temperature regime

Spearman correlations $(p<0.05)$ were observed between PPA concentrations and genera like WCHB1-41 (Kiritimatiellae) or Lentimicrobiaceae (uncultured genus) (Fig. 1).

The LEfSe algorithm was used to search for significant biomarkers. Significant biomarkers with an LDA score of $\geq 4$ for high PAA samples were genera like Sedimentibacter, ADurb.Bin120 (Anaerolineaceae), or Anaerolineaceae (uncultured genus). Significant biomarkers with an LDA score $\geq 4$ for the high PPA concentration group included genera like Tepidanaerobacter, Syntrophomonas, or Anaerosalibacter. A detailed list of significant biomarkers for the high PAA and PPA concentration groups can be found in Table 3. For mesophilic LEfSe biomarkers of low and medium PAA and PPA concentration groups, please refer to Additional file 1: Table S1.

\section{Methanogenic properties}

For a detailed presentation and discussion of the gas properties of mesophilic samples, please refer to Wagner et al. [15]. Methane production was detected in all mesophilic samples. Complex protein samples under medium load conditions showed the highest cumulative methane production. Methane production was considerably restricted in medium-load amino acid samples and in high-load complex protein samples. 14 genera belonging to the phylum Euryarchaeota could be found in mesophilic samples. The mean relative abundance of this phylum ranged from $1.73 \pm 0.27 \%$ in high-load $\mathrm{ME}$ samples on day 14 to $10.8 \pm 1.03 \%$ in medium-load ME samples on day 28. The most dominant methanogenic genera were Methanosarcina spp. and Methanosaeta spp.
(Fig. 2 and Additional file 1: Fig. S4). The genus Methanosarcina was predominant in samples fed with complex proteins under medium load conditions at the end of the incubation period, with a mean relative abundance of $5.61 \pm 0.52 \%$ in Cas and $7.22 \pm 1.55 \%$ in ME samples. By contrast, a relatively high abundance of hydrogenotrophic Methanoculleus spp. (and syntrophic bacterium Tepidanaerobacter spp.) could be observed in Cas samples under high load conditions (Additional file 1: Fig. S4). The mean sequence contribution of Euryarchaeota over all mesophilic sequences was $6.31 \pm 2.47 \%$ in low, $4.98 \pm 2.21 \%$ in medium, and $5.07 \pm 2.11 \%$ in the high PAA concentration groups. On genus level, Methanosarcina spp. and Methanosaeta spp. were highest in low phenyl acid concentration groups (Fig. 2). The genera Methanoculleus and Methanofollis were positively $(p<0.05, \mathrm{~B}-\mathrm{H}$ adjusted) correlated with PPA concentration (Fig. 1), and Methanoculleus spp. was a significant LEfSe biomarker for the high PPA concentration group as shown in Table 3.

\section{Thermophilic communities}

\section{Core microbiome and metagenomic biomarkers}

A detailed description of the core microbiome of all substrates can be found in Table 1. Over all thermophilic samples, the genera Defluviitoga and DTU014 (Clostridia) were part of the core microbiome of each variant. The acetate-oxidising bacterium (SAOB) Syntrophaceticus spp. was part of the core microbiome of samples fed with amino acids (Tryp, Tyr, or Phe), whereas the SAOB Tepidanaerobacter spp. was part of the core microbiome of samples fed with complex proteins. By 


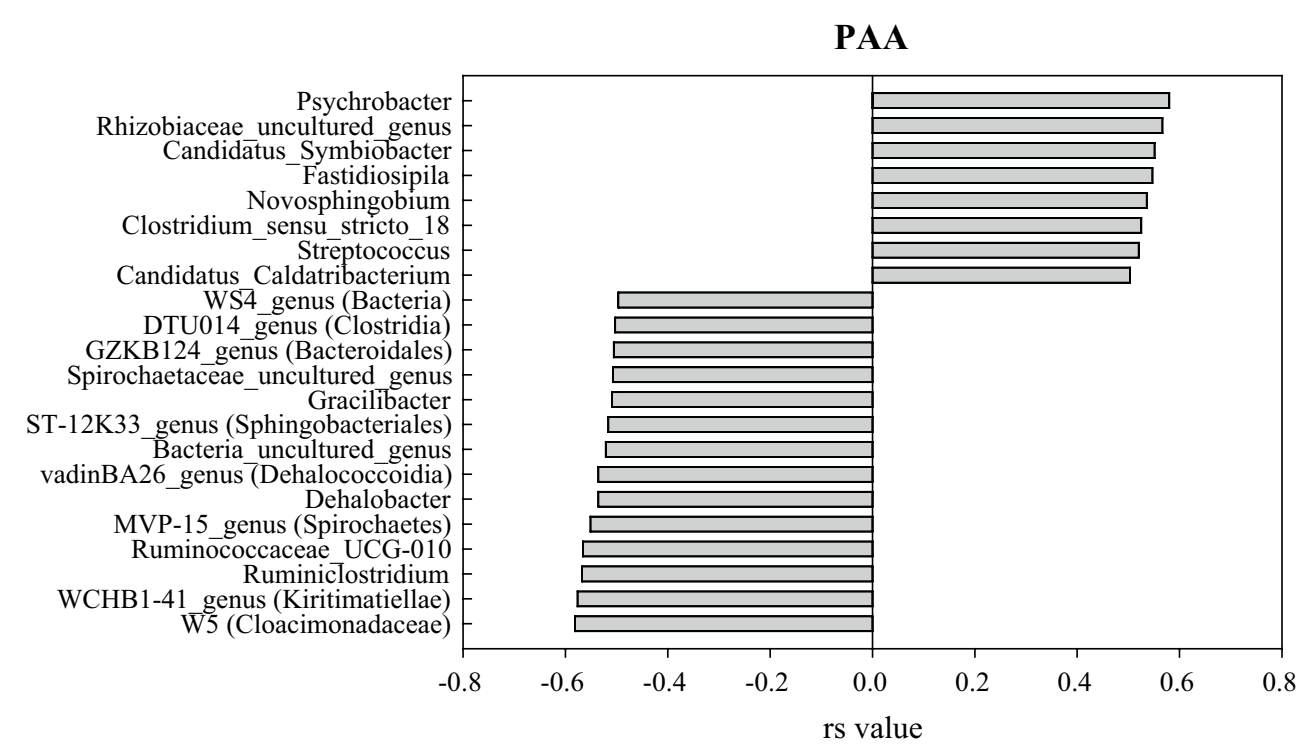

PPA

Haloimpatiens

Proteus

Tepidimicrobium

Asaccharospora

Peptostreptococcus

Saprospiraceae uncultured genus -

Acetomicrobium

Sporanaerobacter

Methanoculleus
Methan

Methanoculleus

Flavobacterium

Eggerthella

Dysgonomonadaceae uncultured genus

Rhizobiaceae_uncultured_genus

Methanofollis

Streptococcus

Clostridium_sensu_stricto_15

Bacteroides

Clostridium sensu stricto 11

Novosphingobium

Defluviimonas

vadinBA26 genus (Dehalococcoidia) -

Bacteroidetes vadinHA17 genus -

Ruminiclostridium

DTU014 genus (Clostridia)

Syner-01 (Synergistaceae)

JS1_genus (Atribacteria)

Armatimonadetes genus

Desulfitobacterium

Armatimonadetes uncultured genus

$$
\text { Ercella }
$$

MVP-15 genus (Spirochaetes)

W5 (Cloacimonadaceae)

Dehalobacter

Bacteria uncultured genus

Paludibacteraceae uncultured genus -

RBG-16-49-21 (Leptospiraceae) -

Hydrogenedensaceae_genus -

WPS-2_genus (Bacteria) Gracilibacter

WS4 genus (Bacteria)

Candidatus Falkowbacteria genus

Spirochaetaceae uncultured genus

Spirochaetaceae uncultured_genus
Lentimicrobiaceae-genus

WCHB1-41_genus (Kiritimatiellae)

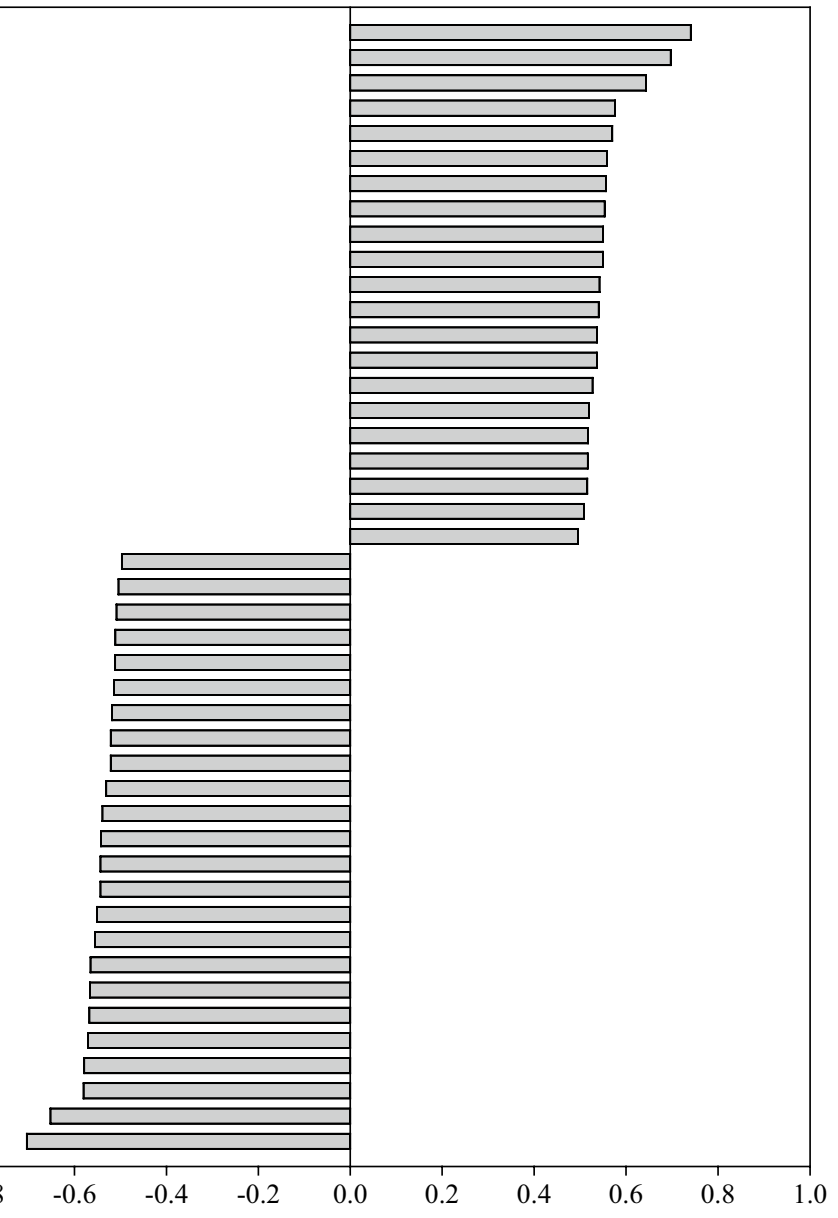

rs value

Fig. 1 Spearman's rank correlation coefficients (rs) including B-H adjustments between mesophilic genera and PAA and between mesophilic genera and PPA concentrations of day 28; Visualisation is restricted to genera with rs values $\leq-0.50$ or $\geq 0.50$. OTU's with a standard deviation $<3$ calculated over all samples were excluded 
Table 3 Meso- (upper row) and thermophilic (lower row) LEfSe biomarker with a LDA score $\geq 4$ of the respective high PAA (left column) or high PPA concentration group (right column)

\begin{tabular}{|c|c|c|c|c|c|c|}
\hline & PAA (class) & Sample size & LEfSe biomarkers & PPA (class) & Sample size & LEfSe biomarkers \\
\hline Mesophilic & High & 12 & $\begin{array}{l}\text { Sedimentibacter } \\
\text { ADurb.Bin120 (Anaerolineaceae) } \\
\text { Anaerolineaceae (uncultured genus) } \\
\text { Tyzzerella } \\
\text { Fastidiosipila } \\
\text { Caproiciproducens } \\
\text { Bacteroidetes_vadinHA17_genus } \\
\text { Candidatus Caldatribacterium } \\
\text { Christensenellaceae_R-7_group } \\
\text { Ruminococcaceae_genus }\end{array}$ & High & 5 & $\begin{array}{l}\text { Tepidanaerobacter } \\
\text { Syntrophomonas } \\
\text { MBA03_genus (Clostridia) } \\
\text { Anaerosalibacter } \\
\text { Firmicutes (uncultured genus) } \\
\text { Terrisporobacter } \\
\text { Proteiniborus } \\
\text { Methanoculleus } \\
\text { Tepidimicrobium } \\
\text { Sporanaerobacter } \\
\text { Clostridiales_FamilyXI (uncultured genus) } \\
\text { SRB2_genus (Clostridia) } \\
\text { Acetomicrobium } \\
\text { Aminobacterium } \\
\text { Ruminococcaceae (uncultured genus) } \\
\text { Fermentimonas }\end{array}$ \\
\hline Thermophilic & High & 9 & $\begin{array}{l}\text { Keratinibaculum } \\
\text { DTU014_genus (Clostridia) } \\
\text { Tepidanaerobacter } \\
\text { Acetomicrobium } \\
\text { Lactobacillus }\end{array}$ & High & 15 & $\begin{array}{l}\text { Sporanaerobacter } \\
\text { Acetomicrobium } \\
\text { Clostridium_sensu_stricto_18 } \\
\text { Sedimentibacter }\end{array}$ \\
\hline
\end{tabular}

Genera in bold are biomarkers in meso- as well as thermophilic samples
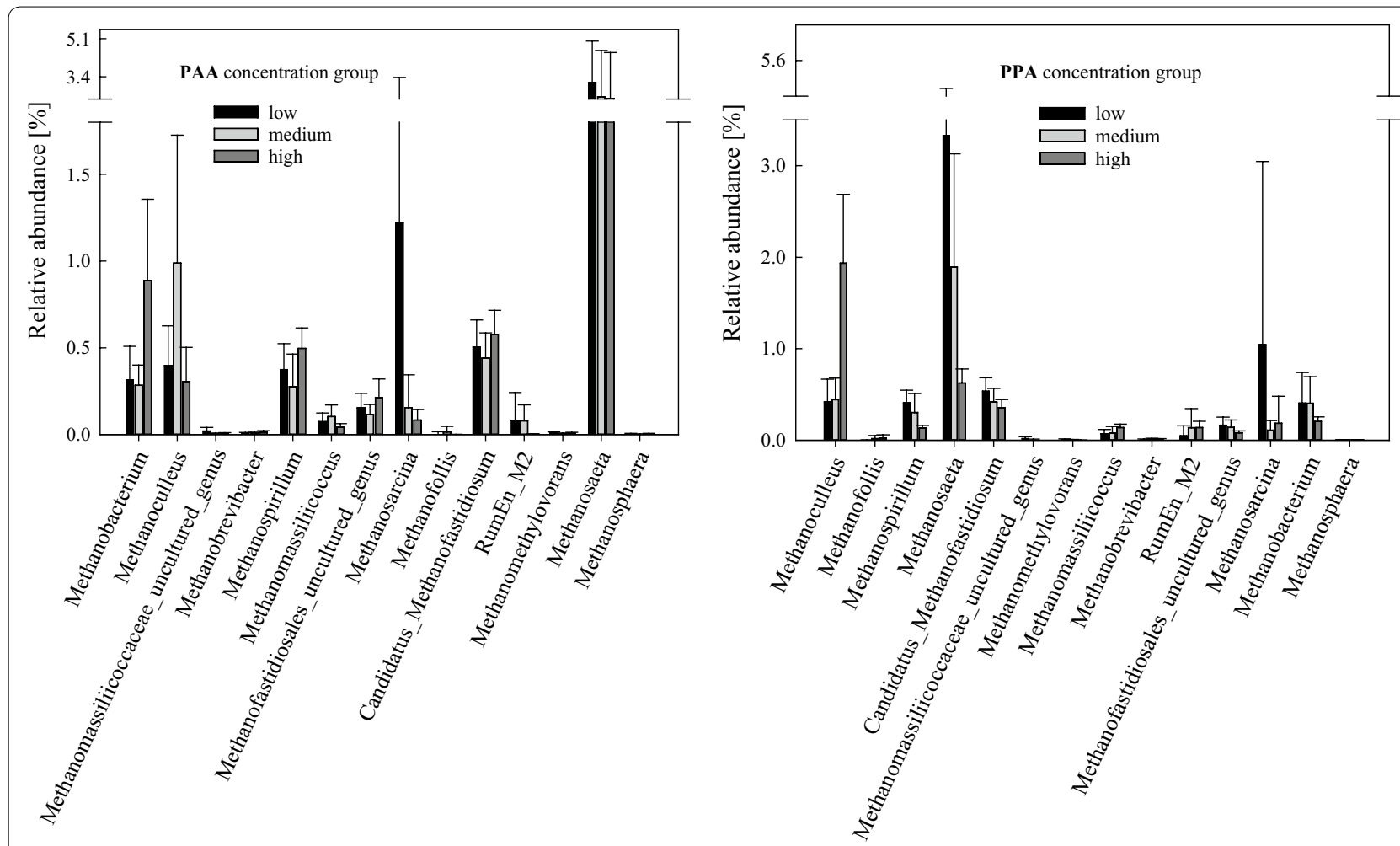

Fig. 2 Relative sequence abundances [\%] of mesophilic methanogens of the low, medium, and high PAA (left) and PPA (right) concentration groups. Bars represent mean values, whiskers standard deviations 
contrast, no SAOB could be found in the core microbiome of the controls (Table 1).

The metagenomic biomarkers (LEfSe analysis, $p<0.05$ ) of all substrate variations are listed in Table 2 . All the biomarkers calculated for samples fed with complex proteins were also part of the core microbiome of the respective samples (Tables 1 and 2).

\section{Phenyl acids and community dynamics}

Results of thermophilic phenyl acid formation were published previously [15] and are presented in a summarised form in Additional file 1: Fig. S6.

Compared with the mesophilic approach, considerably fewer microorganisms correlated with PAA and PPA concentrations. Except for Clostridium_sensu_stricto_18, the genera significantly correlating with phenyl acids during thermophilic incubation were different from those found during mesophilic incubation. PAA concentration positively correlated $(p<0.05)$ with the genera Sedimentibacter, Lactobacillus, Leuconostoc, M55-D21_genus, Syntrophaceticus, Geobacillus, and Corynebacterium_1 (Fig. 3). Additional information on the latter two genera can be looked up in Additional file 1: Text S3. PAA concentration negatively correlated $(p<0.05)$ with the genera Peptococcaceae (uncultured genus) and Proteiniphilum. The genera Sedimentibacter and Syntrophaceticus positively correlated with both PAA and PPA concentration. Moreover, Clostridium_sensu_stricto_18 and Caproiciproducens spp. positively correlated with PPA but not with PAA concentration. No negative $(p<0.05)$ correlations could be found between PPA concentration and thermophilic genera on day 28 .

The genera Sedimentibacter, Tepidanaerobacter, Acetomicrobium, and Sporanaerobacter were significant
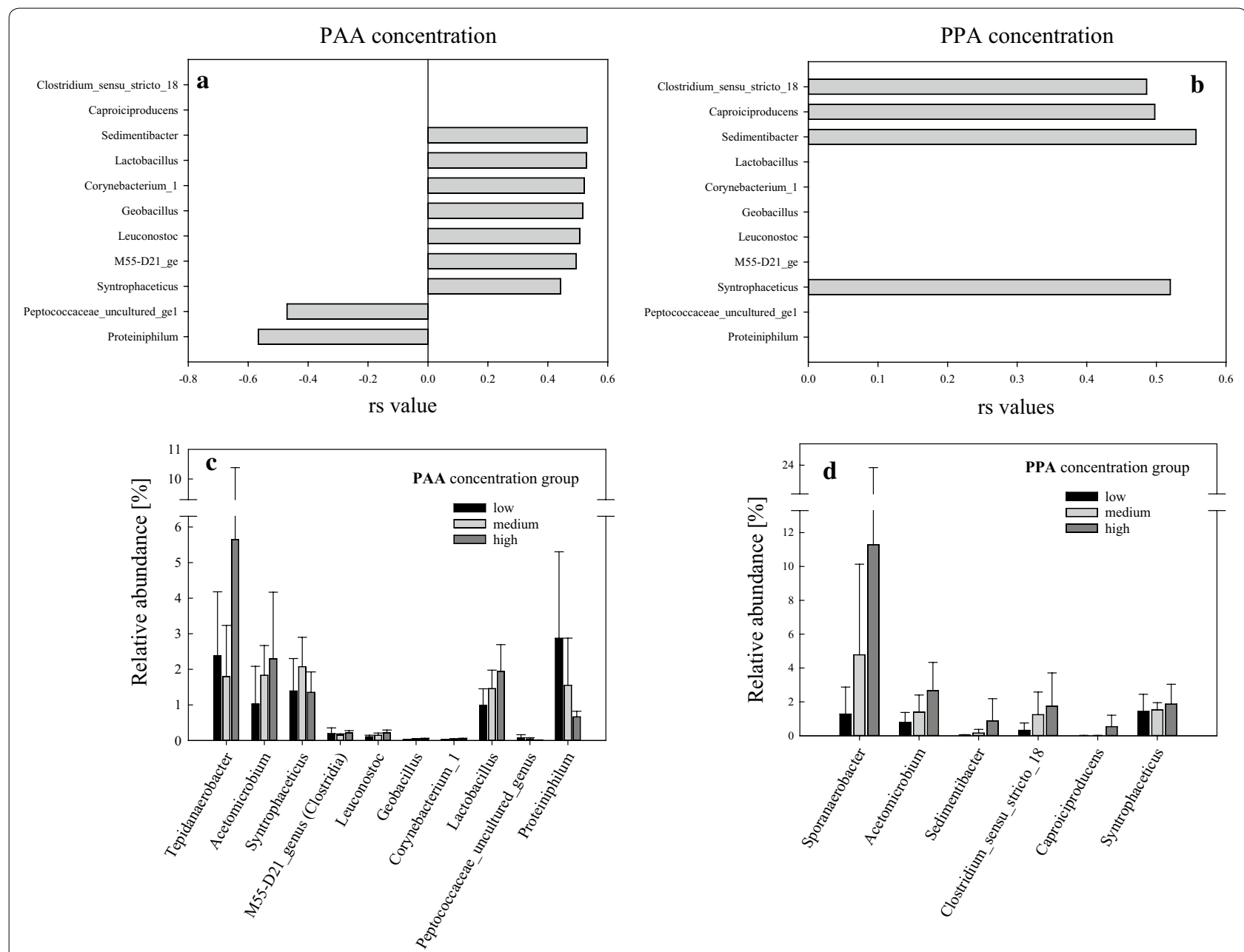

Fig. 3 Spearman's rank correlation coefficients ( $r$ s) including B-H adjustments between thermophilic genera and PAA (a) and between thermophilic genera and PPA (b) concentrations of day 28. OTUs with a standard deviation $<3$ calculated over all samples were excluded. Relative sequence abundances of relevant LEFSe and Spearman genera of low, medium, and high PAA (c) and PPA (d) concentration groups. Bars represent mean values, whiskers the respective standard deviations 
LEfSe biomarkers (LDA $\geq 4)$ for mesophilic as well as thermophilic high phenyl acid concentration groups (Table 3). Moreover, Lactobacillus spp., DTU014_genus, and Keratinibaculum spp. were significant biomarkers for high PAA concentration group. From all the LEfSe biomarkers for high PAA concentration, Tepidanaerobacter spp. showed the highest abundance in the high PAA concentration group: the mean relative abundance ranged from $2.38 \pm 1.80 \%$ in the low to $5.65 \pm 4.74 \%$ in the high PAA concentration group (Fig. 3c). From all the LEfSe biomarkers for high PPA concentration, Sporanaerobacter spp. showed the highest mean relative abundance, ranging from $1.28 \pm 1.59 \%$ in the low to $11.3 \pm 12.5 \%$ in the high PPA concentration group (Fig. 3d). For information on thermophilic LEfSe biomarkers of the low and medium PAA and PPA concentration groups, please refer to Additional file 1: Table S1.

\section{Methanogenic properties}

For a detailed presentation and discussion of the gas properties of all thermophilic samples, please refer to Wagner et al. [15]. Methane production was observed in all thermophilic samples. The highest cumulative methane production was achieved in ME and Cas samples under high overload conditions, whereas the lowest cumulative methane yields could be observed in reactors fed with amino acids under medium overload conditions [15]. When looking at Archaea specifically, eight genera could be assigned to the phylum Euryarchaeota as shown in Fig. 4. The sequences of this phylum contributed with
$0.28 \pm 0.23 \%$ to the low, with $0.30 \pm 0.18 \%$ to the medium, and with $0.67 \pm 0.53 \%$ to the high PAA concentration group and with $0.33 \pm 0.34 \%$ in the low, with $0.39 \pm 0.29 \%$ in the medium, and with $0.23 \pm 0.11 \%$ in the high PPA concentration group. The genera Methanosarcina spp. and Methanothermobacter spp. were the most abundant methanogens in thermophilic controls at day 0 with a sequence contribution of $0.04 \pm 0.02 \%$ and $0.02 \%$, respectively. Over the course of the incubation, Methanoculleus spp. became the most abundant methanogen over all thermophilic samples, followed by Methanothermobacter spp.; the highest abundances of these two genera were shown in the high PAA concentration group (Fig. 4). The relative abundance of Methanosaeta spp., which was very low in general, was even lower at elevated PAA concentrations.

\section{Prediction of metagenomic properties (piphillin)}

The analysis inferred 250 OTUs which exceeded the identity threshold of $97 \%$. Furthermore, 288 genomes and 359 KEGG pathways were observed for this data set, including all mesophilic as well as all thermophilic normalised samples. These numbers are comparable to a previous study focusing on the two meso- and thermophilic methanogenic systems [40].

Generally, the orthology counts for peripheral and central benzoyl-CoA pathways were considerably higher in mesophilic than in thermophilic samples (Fig. 5a, b). The enzyme 6-oxocyclohex-1-ene-1-carbonyl-CoA hydrolase, responsible for the anaerobic benzene ring cleavage
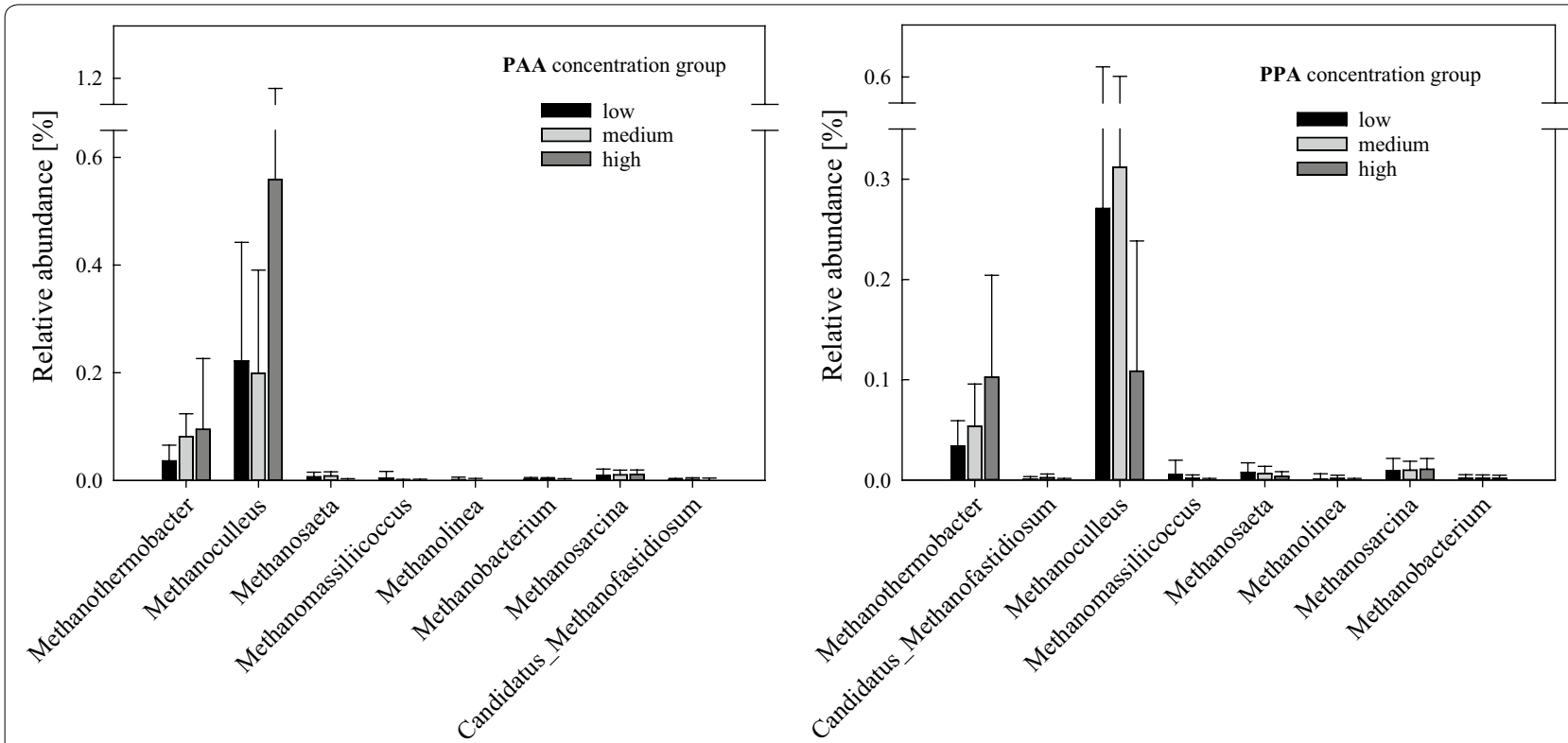

Fig. 4 Relative sequence abundances [\%] of thermophilic methanogens of the low, medium, and high PAA (left) and PPA (right) concentration groups. Bars represent mean values, whiskers standard deviations 

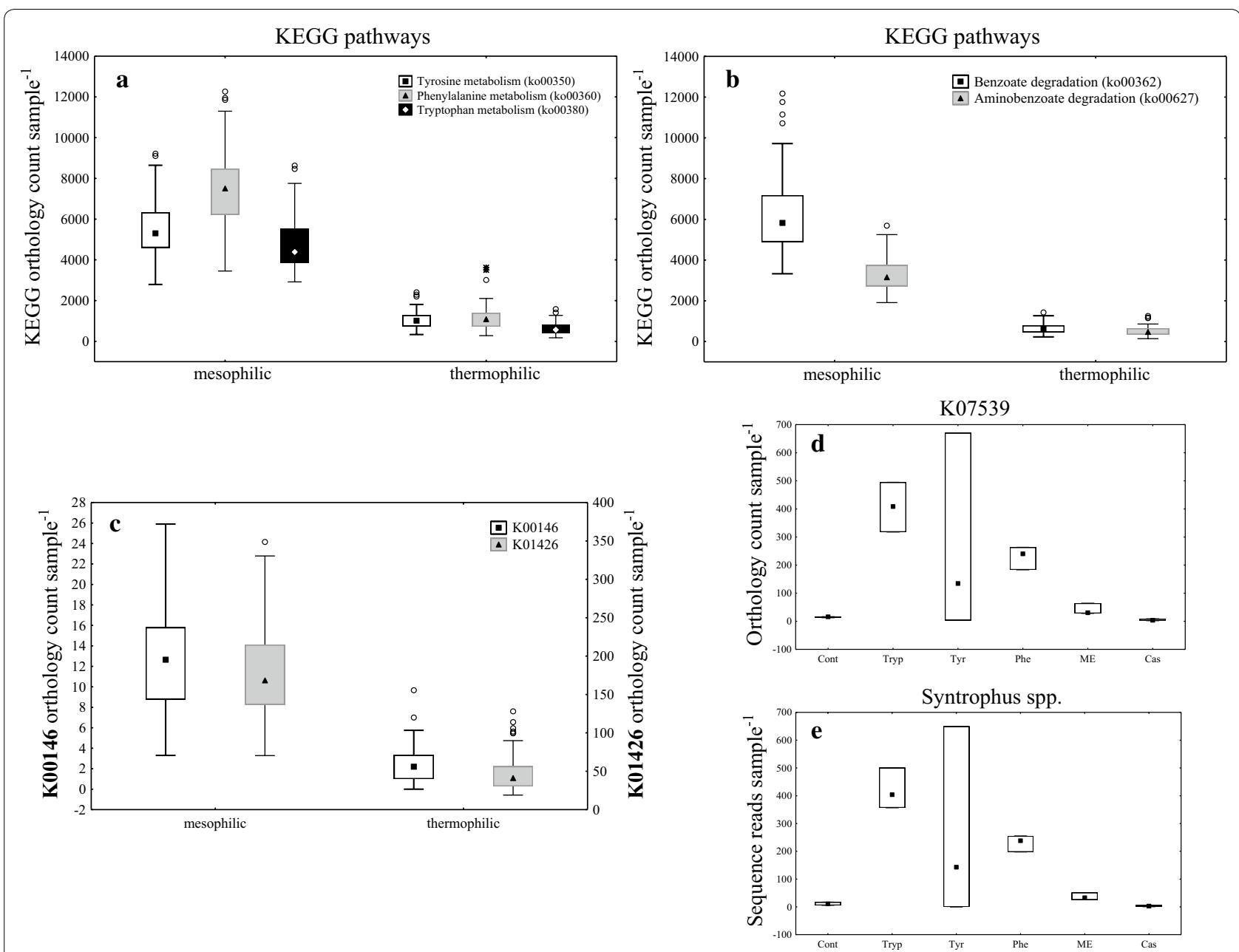

Fig. 5 KEGG orthology counts of amino acid metabolism pathways (a), of (amino-) benzoate degradation pathways (b), and of the enzymes phenylacetaldehyde dehydrogenase (K00146) and amidase (K01426) (c) for all meso- and thermophilic samples. KEGG orthology counts of the enzyme 6-oxocyclohex-1-ene-carbonyl-CoA hydrolase (K07539) of mesophilic samples at low overload conditions on day 28 (d). Sequence abundance of the genus Syntrophus of mesophilic samples at low overload conditions on day 28 (e). The markers represent the median, the boxes show the upper-lower quartiles of each median, the whiskers the non-outlier range (coefficient 1), circles represent outliers, and stars extreme values

during benzoate degradation (KEGG orthology K07539) could only be found in mesophilic samples. More specifically, the enzyme was abundant ( $>60$ orthology counts sample ${ }^{-1}$ ) in Tryp, Tyr, Phe, and ME samples under low overload conditions at day 28 , whereas all other mesophilic variants showed a low abundance. When present in high abundance (Fig. 5d), the orthologue could be assigned to the Syntrophus acidotrophicus genome (Fig. 5e).

The enzyme amidase (K01426), part of the PAA metabolism (Ko00360) and responsible for converting 2-phenylacetamide to PAA, was about tenfold more abundant than the enzyme phenylacetaldehyde dehydrogenase
(K00146), which takes part in forming PAA out of phenylacetaldehyde (Fig. 5c). In mesophilic samples, K01426 could be assigned to the genome Clostridium saccharolyticum WM1 in Tyr samples under medium overload conditions for both day 14 and 28 . The enzyme was also occasionally abundant in Cont, Phe, Cas, and ME samples and could be assigned to the genomes Bradyrhizobium sp. BF49 or Petrimonas sp. IBARAKI (Fig. 5c). In thermophilic samples, the enzyme K01426 was highly abundant in one Phe sample under medium overload conditions at day 28 and could be assigned to Lactobacillus fermentum IFO 3956. 


\section{Discussion}

\section{Mesophilic communities}

The microbial diversity was relatively high in mesophilic samples (Additional file 1: Fig. S3). This is in accordance with previous studies, which showed that mesophilic communities tended to be more diverse and were thus considered less susceptible to disturbances [41, 42]. For further discussions according the microbial diversity of meso- and thermophilic samples, please refer to Additional file 1: Text S1.

Several microorganisms positively correlated with PAA and PPA concentrations (Fig. 1). For instance, the relative abundance of Candidatus Caldatribacterium (about 3\%) and the PAA concentration [15] were highest in medium load Phe samples, which indicates that this microorganism was directly or at least indirectly involved in the conversion of phenylalanine to phenylacetate. This genus belongs to the phylum Atribacteria which is associated with sugar fermentation [43]. Ca. Caldatribacterium was also hypothesised to be acidogenic in thermophilic fermenters fed with Maotai-flavoured distillers' grain, which is characterised by a low $\mathrm{C} / \mathrm{N}$ ratio and a high organic matter content [44].

Phenyl acid degradation was more frequently observed in mesophilic than in thermophilic samples [15]. This is in accordance with Piphillin results, which indicated that the ring cleavage predominantly took place in mesophilic samples (Fig. 5). Ring cleavage could be associated with Syntrophus acidotrophicus (Fig. 5e), a genus that was also a significant biomarker of the low PPA concentration group $(\mathrm{LDA}=3)$ and negatively correlated $(p<0.05)$ with PAA concentrations ( $\mathrm{rs}=-0.46$ ). This indicates its significance for anaerobic benzene ring cleavage. The presence of the genus Syntrophus as well as the enzyme 6-oxocyclohex-1-ene-1-carbonyl-CoA hydrolase were not only restricted to mesophilic but also to low overload samples-irrespective of the substrate used (Additional file 1: Fig. S9). This indicates that high substrate loads can not only lead to higher phenyl acid concentrations in methanogenic systems, but also to a restricted benzene ring cleavage rate. However, this remains to be studied in more detail.

In mesophilic high overload samples, the relative abundances of hydrogenotrophic methanogens and SAOBs were relatively high. This indicates a switch towards SAO-induced hydrogenotrophic methanogenesis; however, a higher utilisation of acetate could not be observed and the methane production was relatively low in these samples [15]. The restricted acetoclastic performance is in accordance with previous studies, which showed that Methanosarcina spp. and especially Methanosaeta spp. are sensitive to typical overload indicators like high ammonium concentrations [8, 45]. The dominance of
Methanosarcina spp. in samples fed with complex proteins at medium overload conditions can be explained by the fact that ammonium concentrations were still relatively low (about $2 \mathrm{~g} \mathrm{NH}_{4}-\mathrm{N} \mathrm{L}^{-1}$ ), while the acetate concentrations were sufficient $(>1 \mathrm{mM})$ during the first 14 days of incubation [8, 15]. Methanosaeta spp. was prevailing especially in low overload samples that were characterised by relatively low acetate and ammonium concentrations [15]. Interestingly, the acetate concentrations were still quite high (about $25 \mathrm{mM}$ on day 0 ) for Methanosaeta spp. to be the dominant acetoclastic methanogen. This indicates that also other biochemical and microbial factors might influence the competitiveness of Methanosaeta spp. Results regarding direct negative effects of phenyl acids on methanogenic Archaea were inconclusive for mesophilic samples. It seems plausible that phenyl acids do not negatively affect all methanogens, but only some representatives of this group. The both negative and positive effects of phenyl acids on methanogens could also be linked to substrate overload conditions. However, this remains to be studied in more detail.

\section{Thermophilic communities}

The microbial diversity in thermophilic samples was considerably lower than in mesophilic samples (Additional file 1: Fig. S3). The dominance (and also importance) of the genus Defluviitoga (phylum Thermotogae), which degrades carbohydrates to $\mathrm{H}_{2} / \mathrm{CO}_{2}$ and acetate, could be confirmed for thermophilic digesters [46-48].

LEfSe analyses showed that Sedimentibacter spp., Tepidanaerobacter spp., Acetomicrobium spp., and Sporanaerobacter spp. were significant biomarkers for both meso- as well as thermophilic phenyl acid formation (Table 3). The LEfSe algorithm is a useful and quite robust three-step tool to analyse metagenomics biomarkers. In this study, it not only elucidated which genera significantly differed between the classes (Kruskal-Wallis $\mathrm{H}$-test), but also considered consistency (Wilcoxon $t$ test) and biological relevance (LDA) [49]. LDA scores of 4 or higher were chosen to highlight the most relevant genera for describing the differences between the classes (and sub-classes). Acetomicrobium hydrogeniformans and $A$. mobile are anaerobic thermophiles known for their ability to degrade Phe; this can lead to an increase in PAA concentration [50-52]. When looking at the organismspecific pathways (Ko00360) in phenylalanine samples, Acetomicrobium (mobile) also contains the enzyme 2-enoate reductase (K10797) responsible for the transformation of trans-cinnamate to PPA. Sporanaerobacter spp. was previously isolated from a pit fermenting strong aromatic liquors at mesophilic temperatures [53]. Tepidanaerobacter spp. oxidises acetate in syntrophic 
association with a hydrogenotrophic methanogenic partner [54]; in the present study, SAO-induced hydrogenotrophic methanogenesis was the most important mineralising process in thermophilic reactors. In the present investigation, the substrate determined whether Syntrophaceticus spp. or Tepidanaerobacter spp. was the dominant SAOB. While Syntrophaceticus spp. was found in Tryp, Tyr, and Phe samples (Additional file 1: Fig. S7), Tepidanaerobacter spp. was found in Cas and ME samples (Table 1, Additional file 1: Fig. S8). The KEGG pathway ko00360 (phenylalanine metabolism) showed that Tepidanaerobacter acetatoxydans was potentially able to degrade 2-phenylacetamide to PAA via an amidase (K01426) [55]. In the present study, thermophilic SAOBs were identified as important players during the degradation of aromatic compounds; however, it remains to be elucidated whether they directly or indirectly contribute to the anaerobic phenyl acid turnover. Further discussion regarding thermophilic SAOBs can be found in Additional file 1: Text S2.

Sedimentibacter spp. further significantly correlated with high PAA concentrations (Fig. 3). Spearman correlation analyses showed that genera like Leuconostoc and Lactobacillus, next to Sedimentibacter spp. and Syntrophaceticus spp., were positively correlated with phenyl acid formation (Fig. 3). Leuconostoc spp. and Lactobacillus spp. belong to the order Lactobacillales and are described as (facultative) anaerobic lactic acid bacteria (LAB) [56-59]. These two genera are normally used as starter organisms in the production of fermented food. They are capable of producing PLA and PAA out of Phe and PLA out of Tyr $[58,60]$. This was primarily described at temperatures around $30{ }^{\circ} \mathrm{C}$. However, also thermophilic LAB exist that convert phenylalanine to PAA during cheese production [60]. Over all thermophilic samples, piphillin analyses showed that a catalase-peroxidase (K03782), responsible for the formation of 2-Phenylacetamide (out of Phe), and an amidase (K01426), responsible for the formation of PAA (out of 2-Phenylacetamide), were more abundant than the enzyme phenylacetaldehyde dehydrogenase (K00146). This indicates that-at least-two different strategies to anaerobically degrade Phe to PAA were possible in thermophilic samples.

Sedimentibacter spp. was not only shown to be involved in phenyl acid formation, but also in anaerobic (amino acid) degradation in meso- and thermophilic systems [61-66]. S. hydroxybenzoicum, isolated from freshwater sediments, was capable of anaerobically degrading phenolic compounds at mesophilic temperatures [65]. The results of this study confirmed that Sedimentibacter spp. is important in the dynamics of aromatic compound formation/degradation during meso- and thermophilic AD. Proteiniphilum spp. and Peptococcaceae (uncultured genus), which negatively correlated with PAA over all thermophilic samples on day 28 (Fig. 3), belong to the phyla Bacteroidetes and Firmicutes, respectively. Even though the family Peptococcaceae is associated with anaerobic benzene degradation [20], the breakdown of PAA and PPA by these microorganisms can be ruled out as their relative abundances were low at high phenyl acid concentrations. The relative abundances of Proteiniphilum spp. were also low at high PAA concentrations (Fig. 3c).

No negative correlations could be found between methanogens and phenyl acid concentrations in thermophilic samples. Thus, these results indicate that methanogens of the thermophilic approach were not impaired by the formed phenyl acids, which is in accordance with the biochemical data previously assessed [15]. When synoptically looking at both meso- and thermophilic reactors, the results implied that the ability of anaerobic phenyl acid formation is not restricted to a certain phylogenetic group of microorganisms but rather wider distributed in the domain Bacteria.

\section{Conclusions}

For both meso- and thermophilic reactors, Sedimentibacter spp., Tepidanaerobacter spp., Acetomicrobium spp., and Sporanaerobacter spp. were shown to be significant biomarkers for high phenyl acid concentrations and thus considered to be involved in the degradation of amino acid and protein-rich precursor substrates. Members of the genus Syntrophus probably took part in the anaerobic benzene ring cleavage in mesophilic samples at low overload conditions (Additional file 1: Fig. S9). They might be important players in preventing phenyl acid accumulation and reactor performance deterioration. Acetoclastic methanogenesis dominated over all mesophilic samples. A shift from acetoclastic to SAO-induced hydrogenotrophic methanogenesis took place in thermophilic samples. This methanogenic pathway seemed to be the quite robust when proteinaceous materials/ precursors were degraded in high loads. Interactions between microbes involved in the formation/degradation dynamics of aromatic compounds were highly complex. Further studies on phenyl acid formation dynamics are thus pending, especially when considering the influence of further factors like temperature, substrate, and substrate load. In further consequence, this knowledge would help to increase the energy exploitation of proteinrich (and lignocellulosic) wastes thus would contribute to a carbon-neutral, economically sustainable, and ethically acceptable energy management. 


\section{Methods}

\section{Experimental setup and sampling}

The samples used in this study derived from an earlier work focusing on the formation of phenyl acids under mesophilic and thermophilic AD conditions [15]. In brief, batch bioreactors contained either Phe, Tyr, Tryp, $\mathrm{ME}$, or Cas as additional substrate. The complex protein substrates $\mathrm{ME}$ and Cas were analysed in three (5.0, 20.0 and $\left.50.0 \mathrm{~g} \mathrm{~L}^{-1}\right)$ and the amino acids Phe, Tyr, and Tryp in two (1.0 and $10.0 \mathrm{~g} \mathrm{~L}^{-1}$ ) different final concentrations. According to the respective substrate load, the samples were grouped into low, medium, and high overload reactors [15]. A control was included containing no additional substrate. Experiments were carried out in triplicates. Samples were incubated at $52{ }^{\circ} \mathrm{C}$ or at $37{ }^{\circ} \mathrm{C}$ for 28 days. Further information on the experimental setup, inocula, lab-use substrates, methane yields, phenyl acid concentrations, and general biochemical properties can be found in the preceding work [15].

Considering the use of two inocula (thus two temperature regimes), three time measuring points, and various substrates at different load conditions [15], 234 samples in total were used for molecular analyses.

\section{DNA extraction}

For molecular biological analyses, $1 \mathrm{~mL}$ samples were taken from each flask after 0,14 , and 28 days. The samples were stored at $-20{ }^{\circ} \mathrm{C}$ until extraction. After thawing, the samples were centrifuged at $20,000 \times g$ for $15 \mathrm{~min}$. Each pellet was washed in $900 \mu \mathrm{L}$ sterile phosphate buffer $(1 \times)$ solution (per litre: $8 \mathrm{~g} \mathrm{NaCl}, 0.2 \mathrm{~g} \mathrm{KCl}, 1.4 \mathrm{~g}$ $\mathrm{Na}_{2} \mathrm{HPO}_{4}, 0.2 \mathrm{~g} \mathrm{KH}_{2} \mathrm{PO}_{4}, \mathrm{pH}$ 7.4), transferred into bead tubes (Macherey-Nagel, Germany) and centrifuged again at $11,000 \times g$ for $10 \mathrm{~min}$. The phosphate buffer was discarded, and DNA extraction was conducted according to the manufacturer's instructions of the Soil Extract II Kit (Macherey-Nagel, Germany). The lysis buffer SL-1 $(700 \mu \mathrm{L})$ and the enhancer $(50 \mu \mathrm{L})$ were added to the washed pellet. Cell lysis took place in a FastPrep- $24^{\mathrm{TM}}$ $5 \mathrm{G}$ (MP Biomedicals, USA) for $1 \times 30 \mathrm{~s}\left(5 \mathrm{~m} \mathrm{~s}^{-1}\right)$. The DNA was eluted in $50 \mu \mathrm{L}$ elution buffer. DNA quantity and quality were measured via NanoDrop 2000c ${ }^{\text {TM }}$ (ThermoFisher Scientific, USA) system. The DNA extracts were diluted to reach a working concentration of $2.5 \mathrm{ng}$ $\mu \mathrm{L}^{-1}$.

\section{NGS library and sequencing}

A simple DNA profiling approach $[67,68]$ was conducted with all variants of day 0 in order to check for the same microbial community structure at the beginning of the experiment. Controls of day 0 and all samples of day 14 and 28 were used for next-generation sequencing (NGS) analyses. The NGS library preparation was conducted in-house. The small subunit rRNA gene primers 515f and 806r [69], according the Earth microbiome project [70], were used to target the V4 region. The first PCR step, including the $16 \mathrm{~S}$ rRNA primers and the Illumina ${ }^{\circledR}$ adapter sequences, was performed as described previously [40]. $25 \mu \mathrm{L}$ PCR solution contained $12 \mu \mathrm{L}$ PCR Mix $\left(\right.$ MyTaq $^{\mathrm{TM}}$ Mix $2 \times$ (Bioline), $250 \mathrm{nM}$ of each primer-adapter combination, 20\% Betaine Enhancer Solution $(5 \times)$ (VWR International, Germany), and PCR-grade water to reach a final volume of $24 \mu \mathrm{L}$, as well as $1 \mu \mathrm{L}$ DNA template (2.5 ng DNA $\left.\mu \mathrm{L}^{-1}\right)$. The quality of the PCR products was checked with a $1.5 \%$ agarose gel. The PCR products of the first step were diluted 1:5 and used as template for a second amplification. For that purpose, the Illumina ${ }^{\circledR}$ barcodes (i5 and i7) were attached. The same PCR procedure as in the first PCR step [40] was conducted except that only five cycles were applied and that the annealing temperature was set to $56{ }^{\circ} \mathrm{C}$. PCR products were again checked with a $1.5 \%$ agarose gel. Subsequently, final PCR products were quantified fluorometrically as described previously [71].

PCR products of each sample (15 ng) were pooled, purified with $\mathrm{Hi}$ Yield $^{\circledR}$ Gel/PCR DNA Fragment Extraction Kit $\left(\mathrm{SLG}^{\circledR}\right.$, Germany), and eluted in $50 \mu \mathrm{L}$ Tris- $\mathrm{HCl}$ buffer. The DNA quantity was again measured via QuantiFluor ${ }^{\circledR}$ dsDNA Dye (Promega, Germany). Co-extraction of contaminants was checked via the NanoDrop 2000c ${ }^{\mathrm{TM}}$ system. The final ready-to-load sample pool showed a DNA concentration of $14 \mathrm{ng}$ $\mu \mathrm{L}^{-1}(260 / 280$ absorbance ratio: 1.88$)$ and was subsequently sent to Microsynth AG in Switzerland where the sequencing was done according to the company's protocols.

\section{Reads procession and OTU classification}

Raw sample reads were processed using the program mothur [72] (v.1.39.5 as well as v.1.42.1 for pre-clustering and chimera search) and the MiSeq SOP (March 2019) [73]. A contig file was created with the paired ends $(10,672,059$ sequences in total, $65,877 \pm 12,374$ sequences sample ${ }^{-1}$ ). After quality filtering (approx. $15 \%$ of the sequences were discarded), unique sequences were aligned to the SILVA V132 database [74]. After another quality check and pre-clustering [75], chimeric amplicons were removed applying the vsearch algorithm (VSEARCH v2.13.3.) [76]. Sequence classification was done with the k-nearest neighbor (knn) algorithm. Sequences were clustered to OTUs based on their taxonomy. For a better comparability of samples while simultaneously ensuring an adequate coverage of the species richness, rarefaction curves were checked, and samples 
were normalised to 19,351 reads sample ${ }^{-1}$. Two samples, both deriving from the thermophilic community on day 14 , were excluded from further analyses due to an insufficient sequencing depth $(n<3060$ sequences per sample). The Mantel test (Gower similarity index) showed that the communities prior to and after rarefaction did not differ significantly $(R=0.94, p<0.01, N=9999)$.

\section{Mock communities}

Three different, defined MOCK communities were included to validate the NGS procedure. The ZymoBI$\mathrm{OMICS}^{\mathrm{TM}}$ Microbial Community standard (Zymo, containing eight bacterial and two yeast microorganisms, further referred to as Mock1) and the archaeon Methanosarcina thermophila DSM 1825 (DSMZ, German Collection of Microorganisms and Cell Cultures, further referred to as Mock2) were analysed separately as well as in combination (50\% genomic DNA Zymo, 50\% genomic DNA M. thermophila, further referred to as Mock3).

The MOCK communities were co-processed with reactor samples. All bacterial and archaeal microorganisms of the three MOCK communities (Mock1, Mock2 and Mock3) could be recovered at genus level. Therefore, the validity and reliability of the applied strategies for DNA extraction, library preparation, and data processing were proven.

\section{Prediction of metagenomic properties}

After subsampling to 19,351 reads per sample, a sequence file containing only representative sequences and an OTU abundance table were generated via mothur (version 1.42.1.). The tool piphillin (https://piphillin. secondgenome.com, July 2019), which uses the nearestneighbor algorithm to pair 16S rRNA gene sequences with genomes [77] was applied. The analyses focused on metagenomic predictions of the peripheral (KEGG orthology ko00350, ko00360, and ko00380) and central (KEGG orthology ko00362 and ko00627) benzoyl-CoA pathways. Moreover, metagenomics prediction of the enzyme 6-oxocyclohex-1-ene-1-carbonyl-CoA hydrolase (KEGG orthology K07539), associated with anaerobic benzene ring cleavage, was also included. The program used USEARCH v8.1.1861 [28] and an identity cut-off of $97 \%$. The KEGG database (version October, 2018) was used as a Ref. [55].

\section{Graphical and statistical analyses}

The Mock community $(n=9)$ check as well as the ecological diversity analyses (Shannon-Weaver index) were done with RStudio $^{\circledR}$ using the packages ggplot 2 and phyloseq [78].

Thenceforth, meso- and thermophilic data were analysed separately; only OTU's with a total abundance of $\geq 10$ were used for each temperature regime (abundance sample ${ }^{-1}$ of removed OTUs: $\leq 5$ ). In mothur, the LEfSe [49] and get.coremicrobiome command were used to further analyse communities on a metagenomic basis. For the general description of biomarkers via LEfSe, the substrate was set as class and the degree of overload (low, medium, high) as subclass. Biomarker discovery for samples with low, medium, and high phenyl acid production was done via $k$-means clustering of PAA and PPA concentrations (low: 0-2.66, medium: 2.74-9.35, and high: 11.9-23.2 mM PAA; low: 0-1.98, medium: 2.07-6.97, and high: 7.56-21.4 mM PPA). The LEfSe algorithm uses the Kruskal-Wallis $\mathrm{H}$-test [79] for detecting biomarkers for the respective class. Within each class, the pairwise Wilcoxon $t$-test [80] is used for detecting biomarkers of subclasses. The LEfSe algorithm also includes linear discriminant analyses (LDA) to further estimate the magnitude of each effect (thus also takes the effect size into consideration) [49].

Spearman correlation analyses (done for samples of day 28), $k$-means clustering, and the Mantel test were done with $P A S T^{\circledR} 3$ [81]. For Spearman's rank correlations coefficient analyses, OTUs showing low variation (standard deviation $<3$ ), calculated over all samples of the respective temperature regime, were excluded to reduce background noise. For correlation analyses, the B-H procedure [82] was applied in Microsoft ${ }^{\circledR}$ Excel $^{\circledR}$. For Spearman's rank correlations, the biochemical and OTU data were $\log (x+1)$ and Box-Cox $(x+1)$ transformed, respectively.

Significant microorganisms for samples with low and high phenyl acid production were processed with the program STAMP 2.1.3 [83]. For that purpose, the White's non-parametric $t$-test (two-sided) was used [84]. When considering effect sizes, genera with a proportion difference below 1 were excluded. Confidence intervals were provided via percentile bootstrapping (1000 permutation test replicates). The false discovery rate was controlled with the $\mathrm{B}-\mathrm{H}$ adjustment.

Graphical presentations of phenyl acid formation and of piphillin analyses were done with Statistica ${ }^{\mathrm{TM}} 13$ (TIBCO ${ }^{\circledR}$ Software Inc.). All other figures were prepared with SigmaPlot ${ }^{\mathrm{TM}} 14$ (Systat $^{\circledR}$ Software Inc). The KRONA tool was used for interactive visualisations of relative sequences abundances [85]. 


\section{Supplementary information}

Supplementary information accompanies this paper at https://doi. org/10.1186/s13068-020-01721-z.

Additional file 1: Table S1. Significant LEFSe biomarker with a LDA score greater or equal 4 for low and medium PAA and PPA concentration groups. Figure S1. Mean sequence proportions [\%] of significant mesophilic phyla of low and high PAA and PPA concentration groups. Figure S2. Mean sequence proportions [\%] of significant thermophilic phyla of low and high PAA and PPA concentration groups. Figure S3. Shannon diversity index for Cont, Tryp, Tyr, Phe, ME, and Cas samples over all measuring time points under low, medium, and high overload conditions. Figure S4. Interactive visualisation of mesophilic taxa of the controls as well as of the Tryp, Tyr, Phe, ME, and Cas samples at low, medium, and high overload conditions on day 28. Figure S5. Interactive visualisation of thermophilic taxa of the controls as well as of the Tryp, Tyr, Phe, ME, and Cas samples at low, medium, and high overload conditions on day 28. Figure S6. Concentrations of PAA and PPA of mesophilic and thermophilic samples on day 0,14 , and 28 . Figure $\mathbf{S 7}$. Relative sequence abundance [\%] of Syntrophaceticus spp. in thermophilic low, medium, and high overload samples on day 28. Figure S8. Relative sequence abundance [\%] of Tepidanaerobacter spp. in thermophilic low, medium, and high overload samples on day 28. Figure S9. Relative sequence abundance [\%] of Syntrophus spp. in mesophilic low, medium, and high overload samples. Text S1. Differences in microbial diversity between meso- and thermophilic communities. Text S2. SAO- induced hydrogenotrophic methanogenesis in thermophilic samples. Text S3. Further positive Spearman correlations between phenyl acid formation and thermophilic genera.

\section{Abbreviations}

SAO: Syntrophic acetate oxidation; Tryp: Tryptophan; Tyr: Tyrosine; Phe: Phenylalanine; PAA: Phenylacetate; PPA: Phenylpropionate; PLA: Phenyllactate; KEGG: Kyoto Encyclopedia of Genes and Genomes; AD: Anaerobic digestion; ME: Meat extract; Cas: Casein; OTU: Operational taxonomic unit; Cont: Control; LDA: Linear discriminant analysis; LEfSe: Linear discriminant analysis effect size; B-H: Benjamini-Hochberg; SAOB: Syntrophic acetate-oxidising bacterium; LAB: (Facultative) anaerobic lactic acid bacteria; Zymo: ZymoBIOMICS ${ }^{\mathrm{TM}}$ Micro- $^{-}$ bial Community standard; DSMZ: German Collection of Microorganisms and Cell Cultures; rs: Spearman's rank correlations coefficient.

\section{Acknowledgements}

Sieglinde Farbmacher is greatly acknowledged for preparing the batch reactors and doing the sampling. We also thank Florian Reischer for the PCR preparations as well as Mira Mutschlechner, Nina Lackner, and Rudolf Markt for their collegial support.

\section{Authors' contributions}

The NGS library was created and checked by EMP and AOW. Reads procession, graphical, statistical, and piphillin analyses as well as writing the manuscript draft were done by EMP. The funding was raised by AOW. AOW, PI and BS supervised the findings of this study. All the authors gave critical feedback and helped shaping analyses and the manuscript. All authors read and approved the final manuscript.

\section{Funding}

The study was supported by the Austrian Science Fund and the county of Tyrol (FWF, Stand-Alone project P29143) as well as the Universität Innsbruck (Publikationsfonds).

BS was in part supported by the CEEPUS Freemover Grant and the LFUI-Guest Professorship of the Universität Innsbruck.

\section{Availability of data and materials}

Mesophilic and thermophilic sequences were uploaded to GenBank ${ }^{\circledR}$ via the submission tool Banklt (BioProject ID for mesophilic samples: 564060, BioProject ID for thermophilic samples: 564063).

\section{Ethics approval and consent to participate}

Not applicable.

\section{Consent for publication}

Not applicable.

\section{Competing interests}

The authors declare that they have no competing interests.

\section{Author details}

${ }^{1}$ Department of Microbiology, Universität Innsbruck, Technikerstraße 25d, 6020 Innsbruck, Austria. ${ }^{2}$ Department of Animal Science, Biotechnical Faculty, University of Ljubljana, Jamnikarjeva 101, 1000 Ljubljana, Slovenia. ${ }^{3}$ Institute of Sanitary Engineering, Faculty of Civil and Geodetic Engineering, University of Ljubljana, Jamova 2, 1000 Ljubljana, Slovenia. ${ }^{4}$ Department of Automation, Biocybernetics and Robotics, Jozef Štefan Institute, Jamova 39, 1000 Ljubljana, Slovenia.

Received: 30 January 2020 Accepted: 24 April 2020

Published online: 06 May 2020

\section{References}

1. Ziemiński K, Romanowska I, Kowalska M. Enzymatic pretreatment of lignocellulosic wastes to improve biogas production. Waste Manag. 2012:32:1131-7.

2. Panwar NL, Kaushik SC, Kothari S. Role of renewable energy sources in environmental protection: a review. Renew Sustain Energy Rev. 2011;15:1513-24

3. Braguglia CM, Gallipoli A, Gianico A, Pagliaccia P. Anaerobic bioconversion of food waste into energy: a critical review. Bioresour Technol. 2018;248:37-56. https://doi.org/10.1016/j.biortech.2017.06.145.

4. Cabrol L, Urra J, Rosenkranz F, Kroff PA, Plugge CM, Lesty Y, Chamy R. Influence of phenylacetic acid pulses on anaerobic digestion performance and archaeal community structure in WWTP sewage sludge digesters. Water Sci Technol. 2015;71:1790. https://doi.org/10.2166/wst.2015.165.

5. Hecht C, Griehl C. Investigation of the accumulation of aromatic compounds during biogas production from kitchen waste. Bioresour Technol. 2009;100:654-8. https://doi.org/10.1016/j.biortech.2008.07.034.

6. Griehl C, Hecht C, Streuber S, Ganß Y, Kremp H. Bestimmung von Phenylessigsäure zur Früherkennung von Prozessstörungen in Biogasanlagen Chem Ing Tec. 2010;82:2223-9. https://doi.org/10.1002/cite.200900064.

7. Qiao J-T, Qiu Y-L, Yuan X-Z, Shi X-S, Xu X-H, Guo R-B. Molecular characterization of bacterial and archaeal communities in a full-scale anaerobic reactor treating corn straw. Bioresour Technol. 2013;143:512-8. https:// doi.org/10.1016/j.biortech.2013.06.014.

8. Westerholm M, Moestedt J, Schnürer A. Biogas production through syntrophic acetate oxidation and deliberate operating strategies for improved digester performance. Appl Energy. 2016;179:124-35. https:// doi.org/10.1016/j.apenergy.2016.06.061.

9. Fotidis IA, Karakashev D, Angelidaki I. The dominant acetate degradation pathway/methanogenic composition in full-scale anaerobic digesters operating under different ammonia levels. Int J Environ Sci Technol. 2014;11:2087-94. https://doi.org/10.1007/s13762-013-0407-9.

10. Moestedt J, Påledal S, Schnürer A, Nordell E. Biogas production from thin stillage on an industrial scale-experience and optimisation. Energies. 2013;6:5642-55. https://doi.org/10.3390/en6115642.

11. Westerholm M, Levén L, Schnürer A. Bioaugmentation of syntrophic acetate-oxidizing culture in biogas reactors exposed to increasing levels of ammonia. Appl Environ Microbiol. 2012;78:7619-25. https://doi. org/10.1128/AEM.01637-12.

12. Angelidaki I, Ahring BK. Anaerobic thermophilic digestion of manure at different ammonia loads: effect of temperature. Water Res. 1994;28:72731. https://doi.org/10.1016/0043-1354(94)90153-8.

13. Rétfalvi T, Tukacs-Hájos A, Szabó P. Effects of artificial overdosing of p-cresol and phenylacetic acid on the anaerobic fermentation of sugar beet pulp. Int Biodeterior Biodegrad. 2013;83:112-8. https://doi. org/10.1016/j.ibiod.2013.05.011.

14. Schink B, Philipp B, Müller J. Anaerobic degradation of phenolic compounds. Naturwissenschaften. 2000;87:12-23. https://doi.org/10.1007/ s001140050002.

15. Wagner AO, Prem EM, Markt R, Kaufmann R, Illmer P. Formation of phenylacetic acid and phenylpropionic acid under different overload 
conditions during mesophilic and thermophilic anaerobic digestion. Biotechnol Biofuels. 2019;12:359. https://doi.org/10.1186/s1306 8-019-1370-6.

16. Fuchs $\mathrm{G}$, Boll M, Heider J. Microbial degradation of aromatic compounds_from one strategy to four. Nat Rev Microbiol. 2011;9:803-16. https://doi.org/10.1038/nrmicro2652.

17. Boll M, Fuchs $G$, Heider J. Anaerobic oxidation of aromatic compounds and hydrocarbons. Curr Opin Chem Biol. 2002;6:604-11.

18. Aklujkar M, Risso C, Smith J, Beaulieu D, Dubay R, Giloteaux L, et al. Anaerobic degradation of aromatic amino acids by the hyperthermophilic archaeon Ferroglobus placidus. Microbiology. 2014;160:2694-709. https://doi.org/10.1099/mic.0.083261-0.

19. Boll M, Löffler C, Morris BEL, Kung JW. Anaerobic degradation of homocyclic aromatic compounds via arylcarboxyl-coenzyme A esters: organisms, strategies and key enzymes. Environ Microbiol. 2014;16:612-27. https:// doi.org/10.1111/1462-2920.12328

20. Vogt $\mathrm{C}$, Kleinsteuber S, Richnow H-H. Anaerobic benzene degradation by bacteria. Microb Biotechnol. 2011;4:710-24. https://doi.org/10.111 1/j.1751-7915.2011.00260.x.

21. Foght J. Anaerobic biodegradation of aromatic hydrocarbons: pathways and prospects. J Mol Microbiol Biotechnol. 2008;15:93-120. https://doi. org/10.1159/000121324.

22. Kato S, Chino K, Kamimura N, Masai E, Yumoto I, Kamagata Y. Methanogenic degradation of lignin-derived monoaromatic compounds by microbial enrichments from rice paddy field soil. Sci Rep. 2015;5:14295. https://doi.org/10.1038/srep14295.

23. Carmona M, Zamarro MT, Blázquez B, Durante-Rodríguez G, Juárez JF, Valderrama JA, et al. Anaerobic catabolism of aromatic compounds: a genetic and genomic view. Microbiol Mol Biol Rev. 2009;73:71-133. https ://doi.org/10.1128/MMBR.00021-08.

24. Rabus R, Boll M, Heider J, Meckenstock RU, Buckel W, Einsle O, et al. Anaerobic microbial degradation of hydrocarbons: from enzymatic reactions to the environment. J Mol Microbiol Biotechnol. 2016;26:5-28. https ://doi.org/10.1159/000443997.

25. Abe-Yoshizumi R, Kamei U, Yamada A, Kimura M, Ichihara S. The evolution of the phenylacetic acid degradation pathway in bacteria. Biosci Biotechnol Biochem. 2004;68:746-8. https://doi.org/10.1271/bbb.68.746.

26. Barker HA. Amino acid degradation by anaerobic bacteria. Annu Rev Biochem. 1981;50:23-40. https://doi.org/10.1146/annurev.bi.50.07018 1.000323.

27. Debnar-Daumler C, Seubert A, Schmitt G, Heider J. Simultaneous involvement of a tungsten-containing aldehyde:ferredoxin oxidoreductase and a phenylacetaldehyde dehydrogenase in anaerobic phenylalanine metabolism. J Bacteriol. 2014;196:483-92. https://doi.org/10.1128/ JB.00980-13.

28. Schneider S, Mohamed MES, Fuchs G. Anaerobic metabolism of I-phenylalanine via benzoyl-CoA in the denitrifying bacterium Thauera aromatica. Arch Microbiol. 1997;168:310-20. https://doi.org/10.1007/s002030050 504.

29. Kuntze K, Shinoda Y, Moutakki H, Mclnerney MJ, Vogt C, Richnow H-H, Boll M. 6-Oxocyclohex-1-ene-1-carbonyl-coenzyme A hydrolases from obligately anaerobic bacteria: characterization and identification of its gene as a functional marker for aromatic compounds degrading anaerobes. Environ Microbiol. 2008;10:1547-56. https://doi.org/10.111 1/j.1462-2920.2008.01570.x.

30. Hosoda A, Kasai Y, Hamamura N, Takahata Y, Watanabe K. Development of a PCR method for the detection and quantification of benzoyl-CoA reductase genes and its application to monitored natural attenuation. Biodegradation. 2005;16:591-601. https://doi.org/10.1007/s1053 2-005-0826-5.

31. Harwood CS, Burchhardt G, Herrmann H, Fuchs G. Anaerobic metabolism of aromatic compounds via the benzoyl-CoA pathway. FEMS Microbiol Rev. 1998;22:439-58. https://doi.org/10.1111/j.1574-6976.1998.tb00380.x.

32. Boll M, Fuchs $G$. Identification and characterization of the natural electron donor ferredoxin and of FAD as a possible prosthetic group of benzoylCoA reductase (dearomatizing), a key enzyme of anaerobic aromatic metabolism. Eur J Biochem. 1998;251:946-54.

33. Breese K, Boll M, Alt-Mörbe J, Schägger H, Fuchs G. Genes coding for the benzoyl-CoA pathway of anaerobic aromatic metabolism in the bacterium Thauera aromatica. Eur J Biochem. 1998;256:148-54.
34. Laempe D, Jahn M, Fuchs G. 6-Hydroxycyclohex-1-ene-1-carbonyl-CoA dehydrogenase and 6-oxocyclohex-1-ene-1-carbonyl-CoA hydrolase, enzymes of the benzoyl-CoA pathway of anaerobic aromatic metabolism in the denitrifying bacterium Thauera aromatica. Eur J Biochem. 1999;263:420-9. https://doi.org/10.1046/j.1432-1327.1999.00504.x.

35. Wischgoll S, Heintz D, Peters F, Erxleben A, Sarnighausen E, Reski R, et al. Gene clusters involved in anaerobic benzoate degradation of Geobacter metallireducens. Mol Microbiol. 2005;58:1238-52. https://doi.org/10.111 1/j.1365-2958.2005.04909.x.

36. Boll M, Fuchs G. Benzoyl-coenzyme A reductase (Dearomatizing), a key enzyme of anaerobic aromatic metabolism. ATP dependence of the reaction, purification and some properties of the enzyme from Thauera aromatica strain K172. Eur J Biochem. 1995;234:921-33. https://doi. org/10.1111/j.1432-1033.1995.921_a.x.

37. Sabra W, Roeske I, Sahm K, Antranikian G, Zeng A-P. High temperature biogas reactors to treat stillage from an industrial bioethanol process: metabolic and microbial characterization. Eng Life Sci. 2015;15:743-50. https://doi.org/10.1002/elsc.201500040.

38. Stack RJ, Hungate RE, Opsahl WP. Phenylacetic acid stimulation of cellulose digestion by Ruminococcus albus 8. Appl Environ Microbiol. 1983;46:539-44.

39. Hungate RE, Stack RJ. Phenylpropanoic acid: growth factor for Ruminococcus albus. Appl Environ Microbiol. 1982;44:79-83.

40. Prem EM, Markt R, Lackner N, Illmer P, Wagner AO. Microbial and phenyl acid dynamics during the start-up phase of anaerobic straw degradation in meso- and thermophilic batch reactors. Microorganisms. 2019;7:657. https://doi.org/10.3390/microorganisms7120657.

41. Sekiguchi Y, Kamagata Y, Syutsubo K, Ohashi A, Harada H, Nakamura K. Phylogenetic diversity of mesophilic and thermophilic granular sludges determined by $16 \mathrm{~S}$ rRNA gene analysis. Microbiology. 1998;144(Pt 9):2655-65. https://doi.org/10.1099/00221287-144-9-2655.

42. Labatut RA, Angenent LT, Scott NR. Conventional mesophilic vs. thermophilic anaerobic digestion: a trade-off between performance and stability? Water Res. 2014;53:249-58. https://doi.org/10.1016/j.watre s.2014.01.035.

43. Dodsworth JA, Blainey PC, Murugapiran SK, Swingley WD, Ross CA, Tringe $\mathrm{SG}$, et al. Single-cell and metagenomic analyses indicate a fermentative and saccharolytic lifestyle for members of the OP9 lineage. Nat Commun. 2013;4:1854. https://doi.org/10.1038/ncomms2884.

44. Ao T, Li R, Chen Y, Li C, Li Z, Liu X, et al. Anaerobic thermophilic digestion of maotai-flavored distiller's grains: process performance and microbial community dynamics. Energy Fuels. 2019;33:8804-11. https://doi. org/10.1021/acs.energyfuels.9b02582.

45. Westerholm M, Dolfing J, Sherry A, Gray ND, Head IM, Schnurer A. Quantification of syntrophic acetate-oxidizing microbial communities in biogas processes. Environ Microbiol Rep. 2011;3:500-5. https://doi.org/10.111 1/j.1758-2229.2011.00249.x.

46. Maus I, Cibis KG, Bremges A, Stolze Y, Wibberg D, Tomazetto G, et al. Genomic characterization of Defluviitoga tunisiensis $L 3$, a key hydrolytic bacterium in a thermophilic biogas plant and its abundance as determined by metagenome fragment recruitment. J Biotechnol. 2016;232:5060. https://doi.org/10.1016/j.jbiotec.2016.05.001.

47. Hania WB, Godbane R, Postec A, Hamdi M, Ollivier B, Fardeau M-L. Defluviitoga tunisiensis gen. nov., sp. nov., a thermophilic bacterium isolated from a mesothermic and anaerobic whey digester. Int J Syst Evol Microbiol. 2012;62:1377-82. https://doi.org/10.1099/ijs.0.033720-0.

48. Westerholm M, Isaksson S, Karlsson Lindsjö O, Schnürer A. Microbial community adaptability to altered temperature conditions determines the potential for process optimisation in biogas production. Appl Energy. 2018;226:838-48. https://doi.org/10.1016/j.apenergy.2018.06.045.

49. Segata N, Izard J, Waldron L, Gevers D, Miropolsky L, Garrett WS, Huttenhower C. Metagenomic biomarker discovery and explanation. Genome Biol. 2011;12:R60.

50. Maune MW, Tanner RS. Description of Anaerobaculum hydrogeniformans sp. nov., an anaerobe that produces hydrogen from glucose, and emended description of the genus Anaerobaculum. Int J Syst Evol Microbiol. 2012;62:832-8. https://doi.org/10.1099/ijs.0.024349-0.

51. Hania WB, Bouanane-Darenfed A, Cayol J-L, Ollivier B, Fardeau M-L. Reclassification of Anaerobaculum mobile, Anaerobaculum thermoterrenum, Anaerobaculum hydrogeniformans as Acetomicrobium mobile comb. nov., Acetomicrobium thermoterrenum comb. nov. and Acetomicrobium 
hydrogeniformans comb. nov., respectively, and emendation of the genus Acetomicrobium. Int J Syst Evol Microbiol. 2016;66:1506-9. https://doi. org/10.1099/ijsem.0.000910.

52. Menes RJ, Muxí L. Anaerobaculum mobile sp. nov., a novel anaerobic, moderately thermophilic, peptide-fermenting bacterium that uses crotonate as an electron acceptor, and emended description of the genus Anaerobaculum. Int J Syst Evol Microbiol. 2002;52:157-64. https://doi. org/10.1099/00207713-52-1-157.

53. Hu X-L, Wang H-Y, Wu Q, Xu Y. Development, validation and application of specific primers for analyzing the clostridial diversity in dark fermentation pit mud by PCR-DGGE. Bioresour Technol. 2014;163:40-7. https://doi. org/10.1016/j.biortech.2014.04.008.

54. Westerholm M, Roos S, Schnürer A. Tepidanaerobacter acetatoxydans sp. nov., an anaerobic, syntrophic acetate-oxidizing bacterium isolated from two ammonium-enriched mesophilic methanogenic processes. Syst Appl Microbiol. 2011;34:260-6. https://doi.org/10.1016/j.syapm .2010.11.018

55. Kanehisa M, Goto S. KEGG: kyoto encyclopedia of genes and genomes. Nucl Acids Res. 2000;28:27-30. https://doi.org/10.1093/nar/28.1.27.

56. Fusco V, Quero GM, Cho G-S, Kabisch J, Meske D, Neve H, et al. The genus Weissella: taxonomy, ecology and biotechnological potential. Front Microbiol. 2015;6:155. https://doi.org/10.3389/fmicb.2015.00155.

57. Li L, Shin S-Y, Lee KW, Han NS. Production of natural antimicrobial compound D-phenyllactic acid using Leuconostoc mesenteroides ATCC 8293 whole cells involving highly active D-lactate dehydrogenase. Lett Appl Microbiol. 2014;59:404-11. https://doi.org/10.1111/lam.12293.

58. Valerio F, Lavermicocca P, Pascale M, Visconti A. Production of phenyllactic acid by lactic acid bacteria: an approach to the selection of strains contributing to food quality and preservation. FEMS Microbiol Lett. 2004;233:289-95. https://doi.org/10.1111/j.1574-6968.2004.tb09494.x.

59. Mu W, Yu S, Zhu L, Jiang B, Zhang T. Production of 3-phenyllactic acid and 4-hydroxyphenyllactic acid by Pediococcus acidilactici DSM 20284 fermentation. Eur Food Res Technol. 2012;235:581-5. https://doi.org/10.1007/ s00217-012-1768-X.

60. Helinck S, Le Bars D, Moreau D, Yvon M. Ability of thermophilic lactic acid bacteria to produce aroma compounds from amino acids. Appl Environ Microbiol. 2004;70:3855-61. https://doi.org/10.1128/ AEM.70.7.3855-3861.2004

61. Imachi H, Sakai S, Kubota T, Miyazaki M, Saito Y, Takai K. Sedimentibacter acidaminivorans sp. nov., an anaerobic, amino-acid-utilizing bacterium isolated from marine subsurface sediment. Int I Syst Evol Microbiol. 2016;66:1293-300. https://doi.org/10.1099/ijsem.0.000878.

62. Kim E, Lee J, Han G, Hwang S. Comprehensive analysis of microbial communities in full-scale mesophilic and thermophilic anaerobic digesters treating food waste-recycling wastewater. Bioresour Technol. 2018;259:442-50. https://doi.org/10.1016/j.biortech.2018.03.079.

63. Bassani I, Kougias PG, Treu L, Angelidaki I. Biogas upgrading via hydrogenotrophic methanogenesis in two-stage continuous stirred tank reactors at mesophilic and thermophilic conditions. Environ Sci Technol. 2015;49:12585-93. https://doi.org/10.1021/acs.est.5b03451.

64. Li Y-F, Nelson MC, Chen P-H, Graf J, Li Y, Yu Z. Comparison of the microbial communities in solid-state anaerobic digestion (SS-AD) reactors operated at mesophilic and thermophilic temperatures. Appl Microbiol Biotechnol. 2015;99:969-80. https://doi.org/10.1007/s00253-014-6036-5.

65. Levén L, Nyberg K, Schnürer A. Conversion of phenols during anaerobic digestion of organic solid waste - A review of important microorganisms and impact of temperature. J Environ Manag. 2012;95:99-103. https://doi. org/10.1016/j.jenvman.2010.10.021.

66. Ueno Y, Tatara M. Microbial population in a thermophilic packed-bed reactor for methanogenesis from volatile fatty acids. Enzyme Microb Technol. 2008;43:302-8. https://doi.org/10.1016/j.enzmictec.2008.04.007.

67. Wagner AO, Lins P, Malin C, Reitschuler C, Illmer P. Impact of protein-, lipid- and cellulose-containing complex substrates on biogas production and microbial communities in batch experiments. Sci Total Environ. 2013;458-460:256-66. https://doi.org/10.1016/j.scitotenv.2013.04.034.
68. Reitschuler C, Lins P, Wagner AO, Illmer P. Cultivation of moonmilk-born non-extremophilic Thaum and Euryarchaeota in mixed culture. Anaerobe. 2014;29:73-9. https://doi.org/10.1016/j.anaerobe.2013.10.002.

69. Apprill A, MCNally S, Parsons R, Weber L. Minor revision to V4 region SSU rRNA 806R gene primer greatly increases detection of SAR11 bacterioplankton. Aquat Microb Ecol. 2015;75:129-37.

70. Gilbert JA, Jansson JK, Knight R. The Earth Microbiome project: successes and aspirations. BMC Biol. 2014;12:69. https://doi.org/10.1186/s1291 5-014-0069-1.

71. Wagner AO, Praeg N, Reitschuler C, IIImer P. Effect of DNA extraction procedure, repeated extraction and ethidium monoazide (EMA)/propidium monoazide (PMA) treatment on overall DNA yield and impact on microbial fingerprints for bacteria, fungi and archaea in a reference soil. Appl Soil Ecol. 2015;93:56-64. https://doi.org/10.1016/j.apsoil.2015.04.005.

72. Schloss PD, Westcott SL, Ryabin T, Hall JR, Hartmann M, Hollister EB, et al. Introducing mothur: open-source, platform-independent, communitysupported software for describing and comparing microbial communities. Appl Environ Microbiol. 2009;75:7537. https://doi.org/10.1128/ AEM.01541-09.

73. Kozich JJ, Westcott SL, Baxter NT, Highlander SK, Schloss PD. Development of a dual-index sequencing strategy and curation pipeline for analyzing amplicon sequence data on the MiSeq illumina sequencing platform. Appl Environ Microbiol. 2013;79:5112. https://doi.org/10.1128/ AEM.01043-13.

74. Quast C, Pruesse E, Yilmaz P, Gerken J, Schweer T, Yarza P, et al. The SILVA ribosomal RNA gene database project: improved data processing and web-based tools. Nucleic Acids Res. 2013;41:D590-6. https://doi. org/10.1093/nar/gks1219.

75. Huse SM, Welch DM, Morrison HG, Sogin ML. Ironing out the wrinkles in the rare biosphere through improved OTU clustering. Environ Microbiol. 2010;12:1889-98. https://doi.org/10.1111/j.1462-2920.2010.02193.x.

76. Rognes T, Flouri T, Nichols B, Quince C, Mahé F. VSEARCH: a versatile open source tool for metagenomics. PeerJ. 2016;4:e2584. https://doi. org/10.7717/peerj.2584.

77. Iwai S, Weinmaier T, Schmidt BL, Albertson DG, Poloso NJ, Dabbagh K, DeSantis TZ. Piphillin: improved prediction of metagenomic content by direct inference from human microbiomes. PLoS ONE. 2016;11:e0166104. https://doi.org/10.1371/journal.pone.0166104.

78. McMurdie PJ, Holmes S. phyloseq: an R package for reproducible interactive analysis and graphics of microbiome census data. PLoS ONE. 2013;8:e61217. https://doi.org/10.1371/journal.pone.0061217.

79. Kruskal WH, Wallis WA. Use of ranks in one-criterion variance analysis. J Am Stat Assoc. 1952;47:583-621. https://doi.org/10.1080/01621 459.1952 .10483441$.

80. Wilcoxon F. Individual comparisons of grouped data by ranking methods. J Econ Entomol. 1946;39:269. https://doi.org/10.1093/jee/39.2.269.

81. Hammer O, Harper D, Ryan P. PAST: paleontological statistics software package for education and data analysis. Palaeontol Electron. 2001;4:9.

82. Benjamini Y, Hochberg Y. Controlling the false discovery rate: a practical and powerful approach to multiple testing. J Roy Stat Soc Ser B. 1995;57:289-300. https://doi.org/10.1111/j.2517-6161.1995.tb02031.x.

83. Parks DH, Tyson GW, Hugenholtz P, Beiko RG. STAMP: statistical analysis of taxonomic and functional profiles. Bioinformatics. 2014;30:3123-4. https ://doi.org/10.1093/bioinformatics/btu494.

84. White JR, Nagarajan N, Pop M. Statistical methods for detecting differentially abundant features in clinical metagenomic samples. PLoS Comput Biol. 2009;5:e1000352. https://doi.org/10.1371/journal.pcbi.1000352.

85. Ondov BD, Bergman NH, Phillippy AM. Interactive metagenomic visualization in a web browser. BMC Bioinform. 2011;12:377. https://doi. org/10.1186/1471-2105-12-385.

\section{Publisher's Note}

Springer Nature remains neutral with regard to jurisdictional claims in published maps and institutional affiliations. 